CRYSTALLOGRAPHIC COMMUNICATIONS

ISSN 2056-9890

\title{
1-[(Anthracen-9-yl)carbonyl]-2,7-dimethoxy- naphthalene: a chain-like structure composed of face-to-face type dimeric molecular aggregates
}

\author{
Siqingaowa, Takehiro Tsumuki, Kazuki Ogata, Noriyuki Yonezawa and Akiko \\ Okamoto*
}

Department of Organic and Polymer Materials Chemistry, Tokyo University of Agriculture \& Technology (TUAT), Koganei, Tokyo 184-8588, Japan. *Correspondence e-mail: aokamoto@cc.tuat.ac.jp

Received 9 October 2016

Accepted 10 November 2016

Edited by H. Ishida, Okayama University, Japan

Keywords: crystal structure; independent molecules; face-to-face type dimeric molecular aggregate; complementary hydrogen bonds; non-coplanarly accumulated aromatic rings arrangement.

CCDC reference: 1516088

Supporting information: this article has supporting information at journals.iucr.org/e

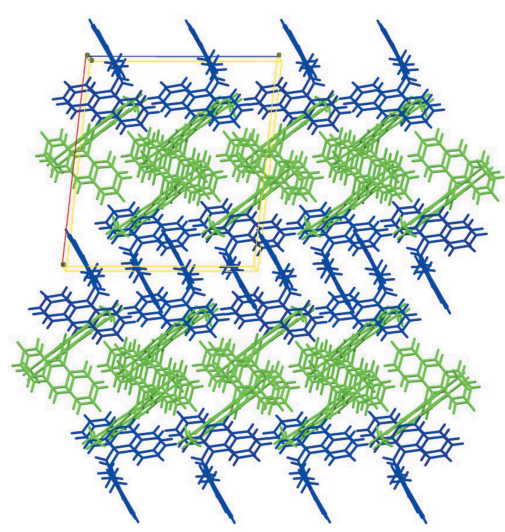

OPEN $\odot$ ACCESS
The asymmetric unit of the title compound, $\mathrm{C}_{27} \mathrm{H}_{20} \mathrm{O}_{3}$, contains two independent molecules $(A$ and $B)$. The anthracene ring system is connected to the 2,7dimethoxynaphthalene core in a twisted manner, with dihedral angles of 86.38 (5) and $79.36(8)^{\circ}$, respectively, for conformers $A$ and $B$. In the crystal, face-to-face type dimeric molecular aggregates of each conformer are observed. The dimer of conformer $A$ is formed by two pairs of $\mathrm{C}-\mathrm{H} \cdots \pi$ interactions, and that of conformer $B$ by a pair of $\left(s p^{2}\right) \mathrm{C}-\mathrm{H} \cdots \mathrm{O}$ hydrogen bonds. The dimers of conformer $A$ are linked to each other via a $\pi-\pi$ stacking interaction between the anthracene rings to form a chain along the $b$ axis and the chains are aligned along the $c$ axis, forming a sheet structure. The dimers of conformer $B$ are connected to each other via a couple of $\mathrm{C}-\mathrm{H} \cdots \pi$ interactions to form a chain along the $b$ axis. The chains are aligned along the $c$ axis through $\left(s p^{2}\right) \mathrm{C}-$ $\mathrm{H} \cdots \mathrm{O}=\mathrm{C}$ hydrogen bonds, forming a sheet parallel to the $b c$ plane. The sheets of conformers $A$ and $B$ are alternately stacked along the $a$ axis via two kinds of intermolecular $\left(s p^{2}\right) \mathrm{C}-\mathrm{H} \cdots \mathrm{O}=\mathrm{C}$ hydrogen bonds.

\section{Chemical context}

Compounds of coplanar aggregation of $\pi$-conjugated aromatic rings have received attention from a wide range of material chemists and organic ones because of their excellent conductivity properties (Lu et al., 2010). Recently, uniquely shaped $\pi$ conjugated aromatic aggregation compounds have moved into the limelight as promising molecular frameworks in nanoelectronics, e.g. bucky bowls (Schmidt et al., 2013), coannulene (Yoshimoto et al., 2010) and cycloparaphenylene (Bunz et al., 2012). These compounds can be regarded as molecules of partial structure and motif of fullerene and carbon nanotubes. On the other hand, aromatic aggregate compounds bearing a non-consecutive $\pi$-conjugated structure have also started to garner attention. For example, the molecular geometry of 9-arylanthracene compounds is of photochemical and photophysical interest because a coplanar spatial arrangement of the anthracene and the aryl substituent $\pi$-systems is precluded due to intramolecular hindrance involving the hydrogen atoms (Becker et al., 1992). In such molecules, the $\pi$-conjugation is weakened and deviations from molecular planarity are borne out in electronic absorption and emission spectra. In particular, the fluorescence spectra of non-coplanarly situated bichromophoric compounds, characterized by large Stokes shifts, are indicative of differences between the geometry of the ground state and that of the more planar emitting excited state (Becker et al., 1990). 


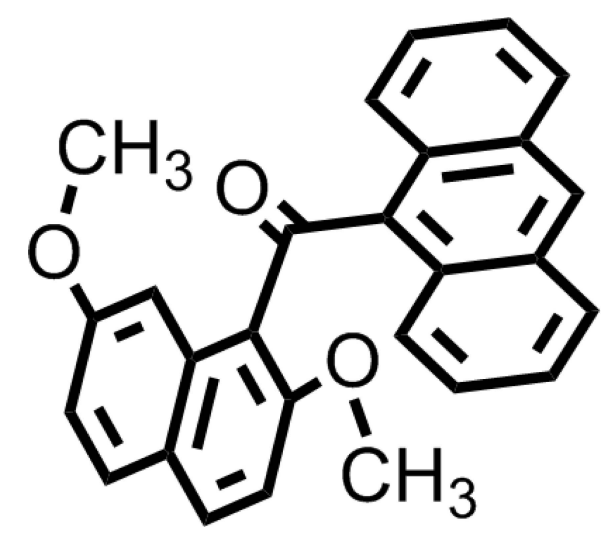

The present authors have studied the synthesis and structure analysis of peri $(1,8)$-aroylated naphthalene compounds in which aromatic rings accumulate non-coplanarly, giving highly congested intramolecular circumstances (Okamoto \& Yonezawa, 2015; Okamoto et al., 2016). As one of the categories of the accumulated $\pi$-conjugated aromatic ring compounds, periaroylnaphthalene compounds have some distinguishable structural characteristics. peri-Aroylnaphthalene compounds belong to the class of poly(aromatic ring) molecules where aromatic rings are linked by ketonic carbonyl groups. Furthermore, the two aroyl groups at peri-positions of the naphthalene ring core are situated in positions very close to each other. Accordingly, a coplanar alignment of the aromatic rings in a molecule is not possible in peri-aroylnaphthalene compounds because of their highly congested molecular arrangement. On the other hand, the spatial organization around the ketonic carbonyl groups of a diaryl ketone structure is supposed to be rather loose compared to that of directly combined aromatic ring systems, as shown in the rotation barrier for an analogous compound in solution
Table 1

Hydrogen-bond geometry $\left(\AA,^{\circ}\right)$.

$C g 1, C g 2$ and $C g 3$ are the centroids of the $\mathrm{C} 20-\mathrm{C} 25, \mathrm{C} 1-\mathrm{C} 5 / \mathrm{C} 10$ and $\mathrm{C} 47-\mathrm{C} 52$ rings, respectively.

\begin{tabular}{lllll}
\hline$D-\mathrm{H} \cdots A$ & $D-\mathrm{H}$ & $\mathrm{H} \cdots A$ & $D \cdots A$ & $D-\mathrm{H} \cdots A$ \\
\hline $\mathrm{C} 7-\mathrm{H} 7 \cdots \mathrm{O} 4^{\mathrm{i}}$ & 0.95 & 2.54 & $3.419(3)$ & 154 \\
$\mathrm{C} 46-\mathrm{H} 46 \cdots \mathrm{O} 1$ & 0.95 & 2.57 & $3.2925(19)$ & 133 \\
$\mathrm{C} 49-\mathrm{H} 49 \cdots \mathrm{O} 4^{\mathrm{ii}}$ & 0.95 & 2.57 & $3.2515(19)$ & 128 \\
$\mathrm{C} 50-\mathrm{H} 50 \cdots \mathrm{O} 6^{\mathrm{iii}}$ & 0.95 & 2.56 & $3.368(2)$ & 143 \\
$\mathrm{C} 3-\mathrm{H} 3 \cdots C g 1^{\text {iv }}$ & 0.95 & 2.68 & $3.557(2)$ & 153 \\
$\mathrm{C} 26-\mathrm{H} 26 A \cdots C 2^{\text {iv }}$ & 0.98 & 2.87 & $3.730(2)$ & 147 \\
$\mathrm{C} 30-\mathrm{H} 30 \cdots C g 3^{\mathrm{v}}$ & 0.95 & 2.71 & $3.602(2)$ & 157 \\
\hline \multicolumn{2}{l}{ Symmetry codes: (i) $x, y-1, z ; \quad$ (ii) } & $x,-y+\frac{1}{2}, z-\frac{1}{2} ; \quad$ (iii) & $-x,-y,-z ;$ (iv) \\
$-x+1,-y+2,-z ;$ (v) $-x,-y+1,-z$. & & &
\end{tabular}

(Okamoto et al., 2012). In this regard, the expected flexibility of the aromatic ketone compound probably shows great variation in the molecular and packing structures in the crystal. Such a situation offers a good opportunity to reveal the hitherto unknown interactions that determine the structure of aromatic rings of accumulated molecules in the crystalline state. This article reports the synthesis and crystal structure of the title 1-anthroylated naphthalene compound.

\section{Structural commentary}

There are two independent molecules in the asymmetric unit of the title compound. The conformers, labeled $A$ and $B$, are shown in Fig. 1. Each conformer has essentially the same noncoplanar structure, with the methoxy group adjacent to the anthroyl group being oriented to the endo side against the naphthalene ring core. The main difference between the conformers is in the orientation of the anthracene ring with respect to the naphthalene ring core. Conformer $A$ shows a dihedral angle of $86.38(5)^{\circ}$ between the anthracene and

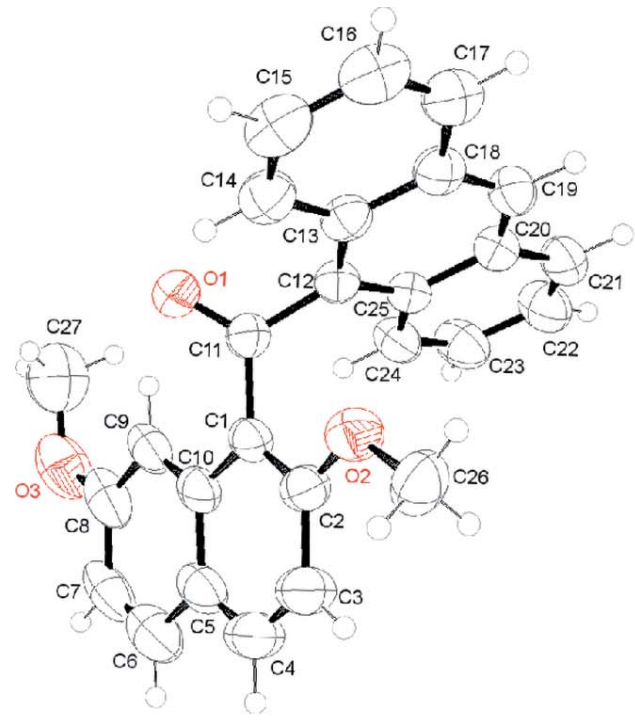

$A$

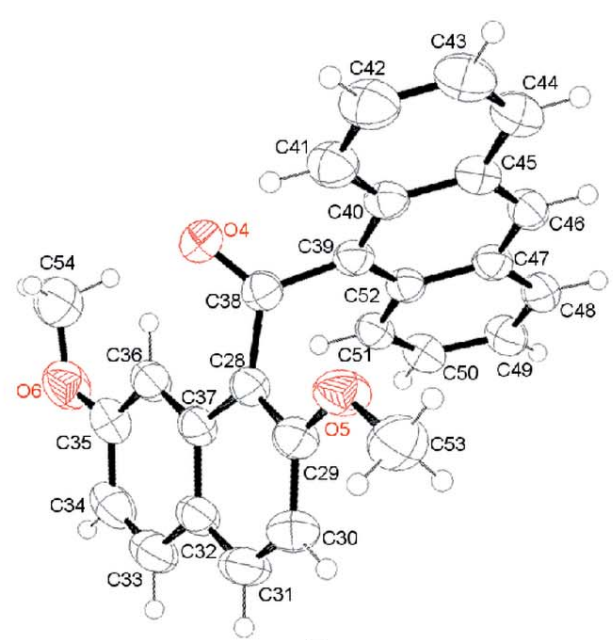

$B$

Figure 1

The structure of the independent molecules $A$ and $B$, showing the atom-labelling scheme. Displacement ellipsoids are drawn at the $50 \%$ probability level for non-H atoms. 


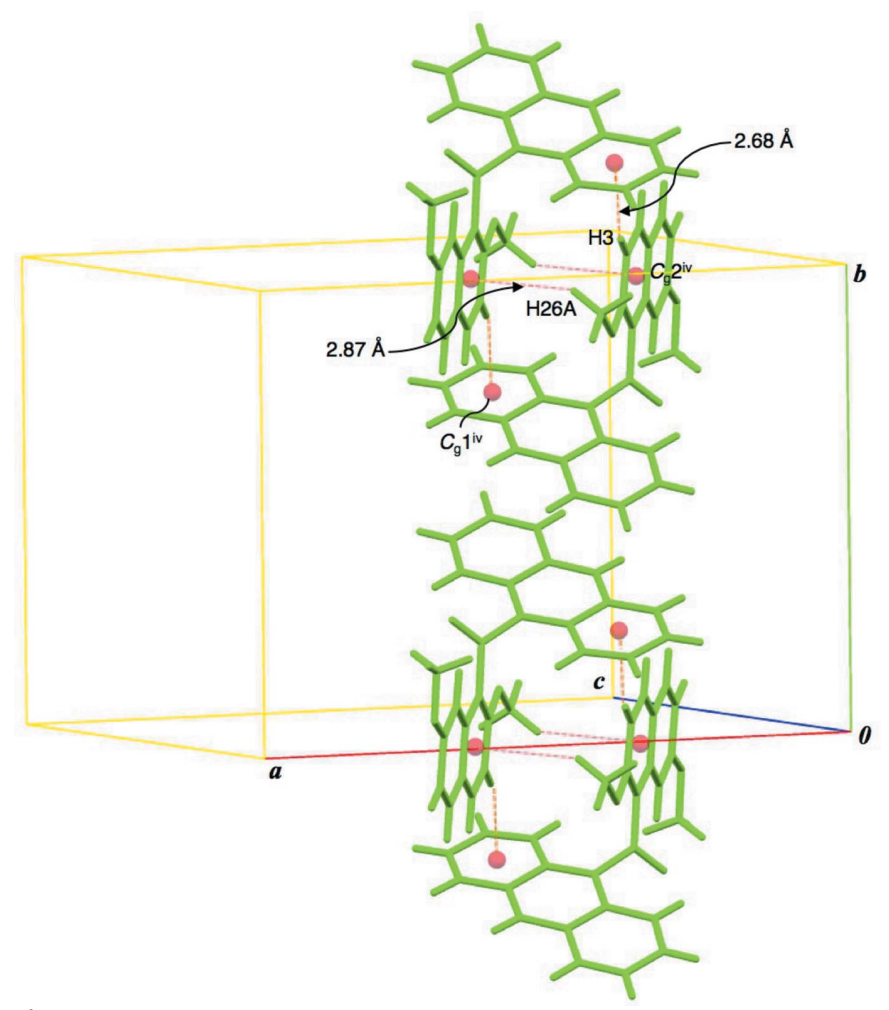

Figure 2

Dimeric molecular aggregates of conformer $A$. Two types of complementary $\mathrm{C}-\mathrm{H} \cdots \pi$ interactions are shown as dashed lines. [Symmetry code: (iv) $-x+1,-y+2,-z$.]

naphthalene ring systems, which is slightly larger than that of $79.36(8)^{\circ}$ for conformer $B$.

\section{Supramolecular features}

Observed non-covalent bonding interactions are summarized in Table 1. In the crystal structure, each conformer forms an inversion dimer with a face-to-face type molecular aggregate by complementary hydrogen bonds. In the dimer of conformer $A$, a pair of (naphthalene) $\mathrm{C}-\mathrm{H} \cdots \pi$ (anthracene) interactions and another pair of (methoxy) $\mathrm{C}-\mathrm{H} \cdots \pi$ (naphthalene) ones are observed $\left(\mathrm{C} 3-\mathrm{H} 3 \cdots C g 1^{\text {iv }}\right.$ and $\mathrm{C} 26-\mathrm{H} 26 A \cdots C g 2^{\text {iv }}$; symmetry code in Table 1; Fig. 2). The dimeric aggregates of conformers $A$ are connected into a chain along the $b$ axis through a $\pi-\pi$ stacking interaction between the anthracene rings [centroid-centroid distance of 3.8198 (10) A between the C12-C13/C18-C20/C25 and C13-C18 rings]. The chains of conformer $A$ are aligned along the $c$ axis by weak van der Waals interactions, forming a sheet structure parallel to the $b c$ plane. In the dimer of conformer $B$, a pair of (anthracene)C$\mathrm{H}$...O(methoxy) hydrogen bonds are observed (C50H50...O66iii; Table 1 and Fig. 3). Furthermore, a pair of (naphthalene) $\mathrm{C}-\mathrm{H} \cdots \pi$ (anthracene) interactions connect the dimeric aggregates into a chain along the $b$ axis (C30H30 $\cdots C g 3^{v}$; Table 1 and Fig. 3). The chains of conformer $B$ are linked by intermolecular (anthracene) $\mathrm{C}-\mathrm{H} \cdots \mathrm{O}=\mathrm{C}$ hydrogen bonds $\left(\mathrm{C} 49-\mathrm{H} 49 \cdots \mathrm{O} 4^{\mathrm{ii}}\right.$; Table 1$)$ along the $c$ axis, forming a sheet parallel to the $b c$ plane. The two sheets of

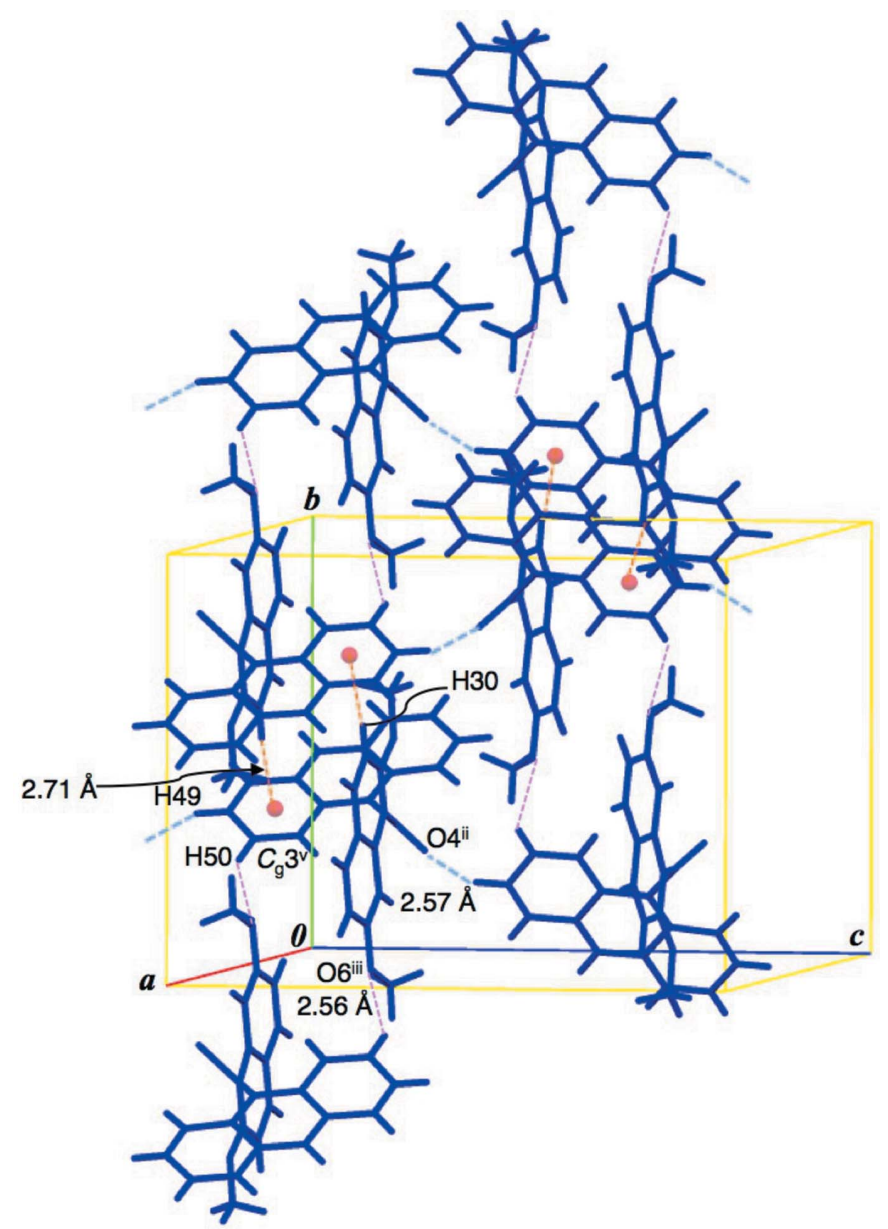

Figure 3

Dimeric molecular aggregates of conformer $B$ and the chain-like molecular alignments. Two kinds of complementary $\mathrm{C}-\mathrm{H} \cdots \pi$ interactions and $\left(s p^{2}\right) \mathrm{C}-\mathrm{H} \cdots$ OMe hydrogen bonds are shown as orange and pink dashed lines. $\mathrm{C}-\mathrm{H} \cdots \mathrm{O}=\mathrm{C}$ hydrogen bonds between the chains are expressed as blue dashed lines. [Symmetry codes: (ii) $x,-y+\frac{1}{2}, z-\frac{1}{2}$; (iii) $-x,-y,-z ;(\mathrm{v})-x,-y+1,-z$.]

conformers $A$ and $B$ are stacked alternately along the $a$ axis by (naphthalene) $\mathrm{C}-\mathrm{H} \cdots \mathrm{O}=\mathrm{C}$ and (anthracene) $\mathrm{C}-\mathrm{H} \cdots \mathrm{O}=\mathrm{C}$ hydrogen bonds $\left(\mathrm{C} 46-\mathrm{H} 46 \cdots \mathrm{O} 1\right.$ and $\mathrm{C} 7-\mathrm{H} 7 \cdots \mathrm{O} 44^{\mathrm{i}}$; Table 1 and Figs. 4 and 5).

\section{Database survey}

A search of the Cambridge Structural Database (CSD Version 5.37, update 2 February 2016; Groom et al., 2016) showed 278 structures of 1-substituted naphthalene compounds containing 1-benzoylated 2,7-dialkoxynaphthalene and 1-naphthoylated 2,7-dialkoxynaphthalene (including $\alpha$-naphthoylated and $\beta$-naphthoylated homologues). The title compound is closely related to (2,7-dimethoxynaphthalen-1-yl)(naphthalen-1-yl)methanone, (I) (Tsumuki et al., 2013), (2,7-dimethoxynaphthalen-1-yl)(phenyl)methanone, (II) (Kato et al., 2010), and 2,7-dimethoxy-1-(2-naphthoyl)naphthalene, (III) (Tsumuki et al., 2012). These homologues have two, three and one independent molecules, respectively, in the asymmetric units of (I), (II) and (III). The dihedral angles between the aromatic ring 


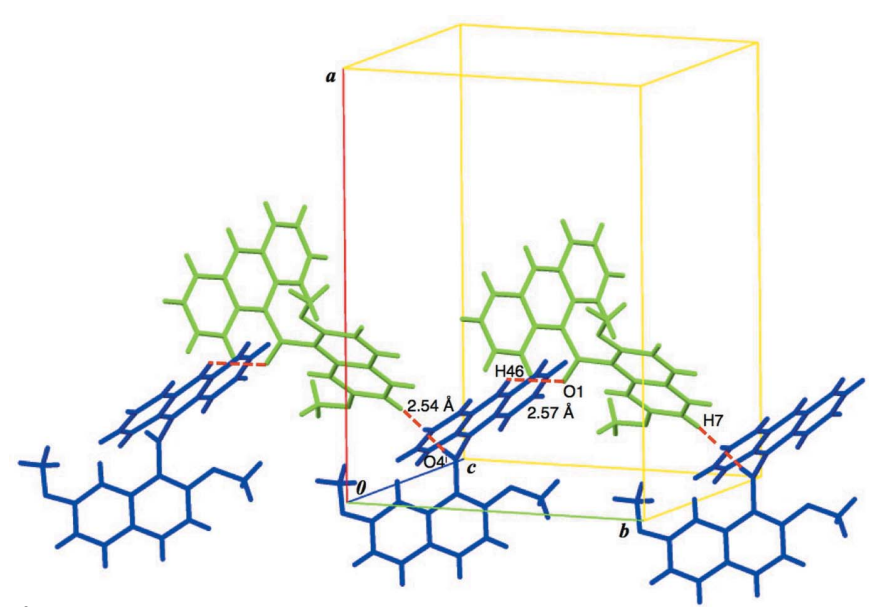

Figure 4

Two kinds of $\left(s p^{2}\right) \mathrm{C}-\mathrm{H} \cdots \mathrm{O}=\mathrm{C}$ hydrogen bonds between conformers $A$ and $B$ are shown as red broken lines. [Symmetry code: (i) $x, y-1, z$.]

of the aroyl group and the 2,7-dimethoxynaphthalene ring core are each 79.07 (4) and $88.19(4)^{\circ}$ for homologue (I), $75.34(7), 86.47(7)$ and $76.55(6)^{\circ}$ for homologue (II), and $80.44(4)^{\circ}$ for homologue (III), which are close to those in the title compound [79.36 (8) and $86.38(5)^{\circ}$ ].

\section{Synthesis and crystallization}

To a solution of 9-anthroyl chloride $(7.8 \mathrm{mmol}, 1.88 \mathrm{~g})$ and $\mathrm{CH}_{2} \mathrm{Cl}_{2}(9.0 \mathrm{~mL}), \mathrm{AlCl}_{3}(7.8 \mathrm{mmol}, 1.04 \mathrm{~g})$ was gradually added. After stirring for $10 \mathrm{~min}$, 2,7-dimethoxynaphthalene ( $3.6 \mathrm{mmol}, 0.67 \mathrm{~g}$ ) was added to the $\mathrm{CH}_{2} \mathrm{Cl}_{2}$ solution. The reaction mixture was stirred in ice-bath for $6 \mathrm{~h}$, then poured

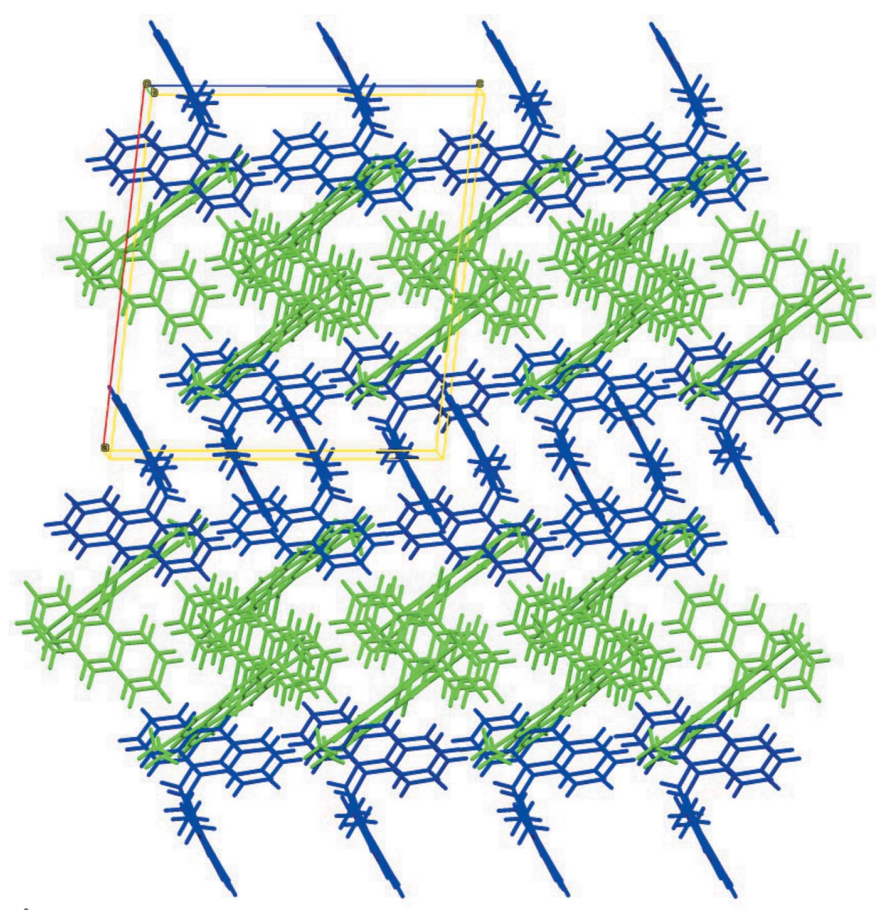

Figure 5

The arrangement of the molecules in the crystal structure, viewed down the $b$ axis.
Table 2

Experimental details.

\begin{tabular}{|c|c|}
\hline \multicolumn{2}{|l|}{ Crystal data } \\
\hline Chemical formula & $\mathrm{C}_{27} \mathrm{H}_{20} \mathrm{O}_{3}$ \\
\hline$M_{\mathrm{r}}$ & 392.43 \\
\hline Crystal system, space group & Monoclinic, $P 2_{1} / c$ \\
\hline Temperature $(\mathrm{K})$ & 193 \\
\hline$a, b, c(\AA)$ & $\begin{array}{l}18.5975(3), 12.9604(2), \\
16.8900(3)\end{array}$ \\
\hline$\beta\left(^{\circ}\right)$ & $96.3650(7)$ \\
\hline$V\left(\AA^{3}\right)$ & $4045.92(12)$ \\
\hline$Z$ & 8 \\
\hline Radiation type & $\mathrm{Cu} K \alpha$ \\
\hline$\mu\left(\mathrm{mm}^{-1}\right)$ & 0.66 \\
\hline Crystal size $(\mathrm{mm})$ & $0.60 \times 0.30 \times 0.10$ \\
\hline \multicolumn{2}{|l|}{ Data collection } \\
\hline Diffractometer & Rigaku R-AXIS RAPID \\
\hline Absorption correction & $\begin{array}{l}\text { Numerical (NUMABS; Higashi, } \\
\text { 1999) }\end{array}$ \\
\hline$T_{\min }, T_{\max }$ & $0.692,0.937$ \\
\hline $\begin{array}{l}\text { No. of measured, independent and } \\
\text { observed }[I>2 \sigma(I)] \text { reflections }\end{array}$ & $59215,7401,4629$ \\
\hline$R_{\text {int }}$ & 0.070 \\
\hline$(\sin \theta / \lambda)_{\max }\left(\AA^{-1}\right)$ & 0.602 \\
\hline \multicolumn{2}{|l|}{ Refinement } \\
\hline$R\left[F^{2}>2 \sigma\left(F^{2}\right)\right], w R\left(F^{2}\right), S$ & $0.040,0.113,0.97$ \\
\hline No. of reflections & 7401 \\
\hline No. of parameters & 546 \\
\hline No. of restraints & 7 \\
\hline $\mathrm{H}$-atom treatment & $\mathrm{H}$-atom parameters constrained \\
\hline$\Delta \rho_{\max }, \Delta \rho_{\min }\left(\mathrm{e} \AA^{-3}\right)$ & $0.31,-0.22$ \\
\hline
\end{tabular}

Computer programs: PROCESS-AUTO (Rigaku, 1998), CrystalStructure (Rigaku, 2010), Il Milione (Burla et al., 2007), SHELXL97 (Sheldrick, 2008) and ORTEPIII (Burnett \& Johnson, 1996).

into ice-cold water. The aqueous layer was extracted with chloroform $(30 \mathrm{ml} \times 3)$ and the combined extracts were washed with $2 M$ aqueous $\mathrm{NaOH}$ solution $(30 \mathrm{ml} \times 3)$ followed by washing with brine $(20 \mathrm{ml} \times 3)$. The organic layer thus obtained was dried over anhydrous $\mathrm{MgSO}_{4}$. The solvent was removed under reduced pressure to give a cake. The title compound was separated from the crude product by column chromatography (eluent: toluene); isolated yield $42 \%$. Yellow needle single crystals suitable for X-ray diffraction were obtained by repeated crystallization from ethyl acetate solution.

${ }^{1} \mathrm{H}$ NMR $\left(500 \mathrm{MHz}, \mathrm{CDCl}_{3}\right) \delta: 3.00(3 \mathrm{H}, s), 3.86(3 \mathrm{H}, s)$, $6.90(1 \mathrm{H}, d, J=9.0 \mathrm{~Hz}), 7.14(1 \mathrm{H}, d d, J=2.5,9.0 \mathrm{~Hz}), 7.42(2 \mathrm{H}$, $d t, J=2.0,7.0 \mathrm{~Hz}), 7.45(2 \mathrm{H}, d t, J=2.0,7.0 \mathrm{~Hz}), 7.76(1 \mathrm{H}, d, J=$ $9.0 \mathrm{~Hz}), 7.87(1 \mathrm{H}, d, J=9.0 \mathrm{~Hz}), 8.0-8.02(4 \mathrm{H}, m), 8.12(1 \mathrm{H}, d$, $J=2.0 \mathrm{~Hz}), 8.49(1 \mathrm{H}, s)$ p.p.m. ${ }^{13} \mathrm{C} \mathrm{NMR}\left(125 \mathrm{MHz}, \mathrm{CDCl}_{3}\right) \delta$ : 55.281, 56.178, 102.94, 111.11, 117.38, 124.07, 124.88, 125.08, $125.46,126.15,128.44,128.51,130.14,131,11,133.42,134.32$, 138.67, 138.76, 159.36, 160.43, 201.25 p.p.m. IR (KBr): $1640(\mathrm{C}=\mathrm{O}), 1618(\mathrm{Ar}), 1511(\mathrm{Ar}), 1250(\mathrm{OMe}) \mathrm{cm}^{-1}$. HRMS $(m / z):[M+\mathrm{H}]^{+}$calculated for $\mathrm{C}_{27} \mathrm{H}_{21} \mathrm{O}_{3}, 393.1491$, found, 393.1494. m.p. $=444.8-446.9 \mathrm{~K}$.

\section{Refinement}

Crystal data, data collection and structure refinement details are summarized in Table 2. All $\mathrm{H}$ atoms were found in a 
difference map and were subsequently refined as riding atoms, with $\mathrm{C}-\mathrm{H}=0.95$ (aromatic) and 0.98 (methyl) $\AA$, and with $U_{\text {iso }}(\mathrm{H})=1.2 U_{\text {eq }}(\mathrm{C})$. Rigid bond restraints $(D E L U)$ were applied to $\mathrm{O} 3-\mathrm{C} 8, \mathrm{C} 1-\mathrm{C} 2, \mathrm{C} 2-\mathrm{C} 3, \mathrm{C} 4-\mathrm{C} 5, \mathrm{C} 5-\mathrm{C} 6, \mathrm{C} 7-$ $\mathrm{C} 8$ and $\mathrm{C} 9-\mathrm{C} 10$ in the naphthalene moiety.

\section{Acknowledgements}

The authors express their gratitude to Professor Keiichi Noguchi, Instrumentation Analysis Center, Tokyo University of Agriculture and Technology, for his technical advice.

\section{References}

Becker, H.-D. (1990). Advances in Photochemistry, Vol. 15, edited by D. H. Volman, G. S. Hammond \& K. Gollinick, p. 139. New York: Wiley \& Sons.

Becker, H.-D., Langer, V., Sieler, J. \& Becker, H.-D. (1992). J. Org. Chem. 57, 1883-1887.

Bunz, U. H. F., Menning, S. \& Martín, N. (2012). Angew. Chem. Int. Ed. 51, 7094-7101.

Burla, M. C., Caliandro, R., Camalli, M., Carrozzini, B., Cascarano, G. L., De Caro, L., Giacovazzo, C., Polidori, G., Siliqi, D. \& Spagna, R. (2007). J. Appl. Cryst. 40, 609-613.
Burnett, M. N. \& Johnson, C. K. (1996). ORTEPIII. Report ORNL6895. Oak Ridge National Laboratory, Tennessee, USA.

Groom, C. R., Bruno, I. J., Lightfoot, M. P. \& Ward, S. C. (2016). Acta Cryst. B72, 171-179.

Higashi, T. (1999). NUMABS. Rigaku Corporation, Tokyo, Japan.

Kato, Y., Nagasawa, A., Hijikata, D., Okamoto, A. \& Yonezawa, N. (2010). Acta Cryst. E66, o2659.

Lu, B., Yan, J., Xu, J., Zhou, S. \& Hu, X. (2010). Macromolecules, 43, 4599-4608.

Okamoto, A., Tsumuki, T., Sasagawa, K., Siqingaowa \& Yonezawa, N. (2016). Eur. Chem. Bull. 5, 211-220.

Okamoto, A., Watanabe, S., Nakaema, K. \& Yonezawa, N. (2012). Cryst. Struct. Theory Appl. 1, 121-127.

Okamoto, A. \& Yonezawa, N. (2015). J. Synth. Org. Chem. Jpn, 73, 339-360.

Rigaku (1998). PROCESS-AUTO. Rigaku Corporation, Tokyo, Japan.

Rigaku (2010). CrystalStructure. Rigaku Corporation, Tokyo, Japan.

Schmidt, B. M., Topolinski, B., Higashibayashi, S., Kojima, T., Kawano, M., Lentz, D. \& Sakurai, H. (2013). Chem. Eur. J. 19, 3282-3286.

Sheldrick, G. M. (2008). Acta Cryst. A64, 112-122.

Tsumuki, T., Isogai, A., Kawano, H., Yonezawa, N. \& Okamoto, A. (2013). Acta Cryst. E69, o663.

Tsumuki, T., Isogai, A., Nagasawa, A., Okamoto, A. \& Yonezawa, N. (2012). Acta Cryst. E68, o2595.

Yoshimoto, S. \& Kobayashi, N. (2010). Struct. Bonding (Berlin), 135, 137-168. 


\section{supporting information}

Acta Cryst. (2016). E72, 1819-1823 [https://doi.org/10.1107/S2056989016018077]

\section{1-[(Anthracen-9-yl)carbonyl]-2,7-dimethoxynaphthalene: a chain-like structure composed of face-to-face type dimeric molecular aggregates}

\section{Siqingaowa, Takehiro Tsumuki, Kazuki Ogata, Noriyuki Yonezawa and Akiko Okamoto}

Computing details

Data collection: PROCESS-AUTO (Rigaku, 1998); cell refinement: PROCESS-AUTO (Rigaku, 1998); data reduction: CrystalStructure (Rigaku, 2010); program(s) used to solve structure: Il Milione (Burla et al., 2007); program(s) used to refine structure: SHELXL97 (Sheldrick, 2008); molecular graphics: ORTEPIII (Burnett \& Johnson, 1996); software used to prepare material for publication: SHELXL97 (Sheldrick, 2008).

1-[(Anthracen-9-yl)carbonyl]-2,7-dimethoxynaphthalene

Crystal data

$\mathrm{C}_{27} \mathrm{H}_{20} \mathrm{O}_{3}$

$M_{r}=392.43$

Monoclinic, $P 2_{1} / c$

Hall symbol: -P 2ybc

$a=18.5975(3) \AA$

$b=12.9604(2) \AA$

$c=16.8900(3) \AA$

$\beta=96.3650(7)^{\circ}$

$V=4045.92(12) \AA^{3}$

$Z=8$

\section{Data collection}

Rigaku R-AXIS RAPID diffractometer

Radiation source: rotating anode

Graphite monochromator

Detector resolution: 10.00 pixels $\mathrm{mm}^{-1}$

$\omega$ scans

Absorption correction: numerical

(NUMABS; Higashi, 1999)

$T_{\min }=0.692, T_{\max }=0.937$

Refinement

Refinement on $F^{2}$

Least-squares matrix: full

$R\left[F^{2}>2 \sigma\left(F^{2}\right)\right]=0.040$

$w R\left(F^{2}\right)=0.113$

$S=0.97$

7401 reflections

546 parameters

7 restraints
$F(000)=1648$

$D_{\mathrm{x}}=1.289 \mathrm{Mg} \mathrm{m}^{-3}$

Melting point $=444.8-446.9 \mathrm{~K}$

$\mathrm{Cu} K \alpha$ radiation, $\lambda=1.54187 \AA$

Cell parameters from 45896 reflections

$\theta=3.4-68.2^{\circ}$

$\mu=0.66 \mathrm{~mm}^{-1}$

$T=193 \mathrm{~K}$

Platelet, yellow

$0.60 \times 0.30 \times 0.10 \mathrm{~mm}$

59215 measured reflections

7401 independent reflections

4629 reflections with $I>2 \sigma(I)$

$R_{\text {int }}=0.070$

$\theta_{\max }=68.2^{\circ}, \theta_{\min }=4.2^{\circ}$

$h=-22 \rightarrow 22$

$k=-15 \rightarrow 15$

$l=-20 \rightarrow 20$

Primary atom site location: structure-invariant direct methods

Secondary atom site location: difference Fourier map

Hydrogen site location: inferred from neighbouring sites

$\mathrm{H}$-atom parameters constrained 
$w=1 /\left[\sigma^{2}\left(F_{\mathrm{o}}^{2}\right)+(0.0601 P)^{2}\right]$

where $P=\left(F_{\mathrm{o}}^{2}+2 F_{\mathrm{c}}^{2}\right) / 3$

$(\Delta / \sigma)_{\max }<0.001$

$\Delta \rho_{\max }=0.31 \mathrm{e} \AA^{-3}$
$\Delta \rho_{\min }=-0.22$ e $\AA^{-3}$

Extinction correction: SHELXL97 (Sheldrick, 2008), $\mathrm{Fc}^{*}=\mathrm{kFc}\left[1+0.001 \mathrm{xFc}^{2} \lambda^{3} / \sin (2 \theta)\right]^{-1 / 4}$

Extinction coefficient: 0.00184 (13)

\section{Special details}

Geometry. All esds (except the esd in the dihedral angle between two 1.s. planes) are estimated using the full covariance matrix. The cell esds are taken into account individually in the estimation of esds in distances, angles and torsion angles; correlations between esds in cell parameters are only used when they are defined by crystal symmetry. An approximate (isotropic) treatment of cell esds is used for estimating esds involving l.s. planes.

Refinement. Refinement of $\mathrm{F}^{2}$ against ALL reflections. The weighted R-factor $\mathrm{wR}$ and goodness of fit $\mathrm{S}$ are based on $\mathrm{F}^{2}$, conventional $R$-factors $R$ are based on $F$, with $F$ set to zero for negative $F^{2}$. The threshold expression of $F^{2}>2 \operatorname{sigma}\left(\mathrm{F}^{2}\right)$ is used only for calculating R-factors(gt) etc. and is not relevant to the choice of reflections for refinement. R-factors based on $\mathrm{F}^{2}$ are statistically about twice as large as those based on F, and R- factors based on ALL data will be even larger.

Fractional atomic coordinates and isotropic or equivalent isotropic displacement parameters $\left(\AA^{2}\right)$

\begin{tabular}{|c|c|c|c|c|}
\hline & $x$ & $y$ & $z$ & $U_{\text {iso }} * / U_{\text {eq }}$ \\
\hline $\mathrm{O} 1$ & $0.30526(6)$ & $0.71806(9)$ & $0.04126(8)$ & 0.0649 (4) \\
\hline $\mathrm{O} 2$ & $0.43048(7)$ & $0.88098(9)$ & $-0.07348(7)$ & $0.0650(4)$ \\
\hline $\mathrm{O} 3$ & $0.19308(8)$ & $0.91363(15)$ & $0.23930(9)$ & $0.0919(5)$ \\
\hline $\mathrm{O} 4$ & $0.10679(6)$ & $0.23802(9)$ & $0.22905(6)$ & $0.0524(3)$ \\
\hline $\mathrm{O} 5$ & $0.03957(6)$ & $0.49125(9)$ & $0.15600(7)$ & $0.0621(3)$ \\
\hline O6 & $-0.03644(7)$ & $-0.05271(10)$ & $0.09642(8)$ & $0.0709(4)$ \\
\hline $\mathrm{C} 1$ & $0.35478(8)$ & $0.88485(13)$ & $0.02904(10)$ & $0.0504(4)$ \\
\hline $\mathrm{C} 2$ & $0.39295(9)$ & $0.93766(14)$ & $-0.02605(11)$ & $0.0551(4)$ \\
\hline $\mathrm{C} 3$ & $0.38780(11)$ & $1.04614(15)$ & $-0.03283(13)$ & $0.0708(6)$ \\
\hline $\mathrm{H} 3$ & 0.4131 & 1.0819 & -0.0703 & $0.085 *$ \\
\hline $\mathrm{C} 4$ & $0.34683(12)$ & $1.09827(16)$ & $0.01425(13)$ & $0.0748(6)$ \\
\hline $\mathrm{H} 4$ & 0.3440 & 1.1712 & 0.0088 & $0.090 *$ \\
\hline $\mathrm{C} 5$ & $0.30795(10)$ & $1.05116(15)$ & $0.07093(12)$ & $0.0655(5)$ \\
\hline C6 & $0.26594(12)$ & $1.10606(18)$ & $0.11951(15)$ & $0.0829(7)$ \\
\hline H6 & 0.2635 & 1.1790 & 0.1139 & $0.099 *$ \\
\hline $\mathrm{C} 7$ & $0.22837(12)$ & $1.06117(19)$ & $0.17422(14)$ & $0.0807(7)$ \\
\hline $\mathrm{H} 7$ & 0.2002 & 1.1012 & 0.2064 & $0.097 *$ \\
\hline $\mathrm{C} 8$ & $0.23233(11)$ & $0.95147(18)$ & $0.18221(12)$ & $0.0741(5)$ \\
\hline C9 & $0.27245(9)$ & $0.89312(15)$ & $0.13665(10)$ & $0.0589(5)$ \\
\hline H9 & 0.2746 & 0.8204 & 0.1438 & $0.071 *$ \\
\hline $\mathrm{C} 10$ & $0.31102(9)$ & $0.94020(14)$ & $0.07859(11)$ & $0.0564(4)$ \\
\hline $\mathrm{C} 11$ & $0.35854(9)$ & $0.77030(13)$ & $0.03250(10)$ & $0.0483(4)$ \\
\hline $\mathrm{C} 12$ & $0.42958(8)$ & $0.71828(12)$ & $0.02502(10)$ & $0.0446(4)$ \\
\hline $\mathrm{C} 13$ & $0.43721(9)$ & $0.65382(13)$ & $-0.04057(9)$ & $0.0475(4)$ \\
\hline $\mathrm{C} 14$ & $0.37884(10)$ & $0.62973(14)$ & $-0.10021(10)$ & $0.0583(5)$ \\
\hline $\mathrm{H} 14$ & 0.3324 & 0.6589 & -0.0969 & $0.070 *$ \\
\hline $\mathrm{C} 15$ & $0.38916(12)$ & $0.56528(15)$ & $-0.16185(11)$ & $0.0671(5)$ \\
\hline H15 & 0.3496 & 0.5491 & -0.2004 & $0.081 *$ \\
\hline $\mathrm{C} 16$ & $0.45763(12)$ & $0.52246(16)$ & $-0.16906(11)$ & $0.0676(5)$ \\
\hline H16 & 0.4636 & 0.4771 & -0.2120 & $0.081 *$ \\
\hline $\mathrm{C} 17$ & $0.51455(11)$ & $0.54500(15)$ & $-0.11592(11)$ & $0.0614(5)$ \\
\hline
\end{tabular}




\begin{tabular}{|c|c|c|c|c|}
\hline H17 & 0.5605 & 0.5161 & -0.1222 & $0.074 *$ \\
\hline $\mathrm{C} 18$ & $0.50708(9)$ & $0.61207(13)$ & $-0.04985(10)$ & $0.0497(4)$ \\
\hline $\mathrm{C} 19$ & $0.56517(9)$ & $0.63494(13)$ & $0.00600(10)$ & $0.0536(4)$ \\
\hline H19 & 0.6118 & 0.6102 & -0.0022 & $0.064 *$ \\
\hline $\mathrm{C} 20$ & $0.55731(9)$ & $0.69286(13)$ & $0.07348(10)$ & $0.0499(4)$ \\
\hline $\mathrm{C} 21$ & $0.61593(10)$ & 0.70988 (14) & $0.13398(12)$ & $0.0618(5)$ \\
\hline $\mathrm{H} 21$ & 0.6627 & 0.6852 & 0.1262 & $0.074 *$ \\
\hline $\mathrm{C} 22$ & $0.60641(11)$ & $0.76003(15)$ & $0.20167(12)$ & $0.0706(6)$ \\
\hline $\mathrm{H} 22$ & 0.6462 & 0.7701 & 0.2413 & $0.085^{*}$ \\
\hline $\mathrm{C} 23$ & $0.53746(11)$ & $0.79769(15)$ & $0.21381(11)$ & $0.0668(5)$ \\
\hline $\mathrm{H} 23$ & 0.5307 & 0.8308 & 0.2626 & $0.080 *$ \\
\hline $\mathrm{C} 24$ & $0.48051(9)$ & $0.78739(13)$ & $0.15684(10)$ & $0.0549(5)$ \\
\hline $\mathrm{H} 24$ & 0.4350 & 0.8158 & 0.1656 & $0.066^{*}$ \\
\hline $\mathrm{C} 25$ & $0.48758(8)$ & $0.73474(12)$ & $0.08406(10)$ & $0.0450(4)$ \\
\hline $\mathrm{C} 26$ & $0.47145(10)$ & $0.93094(16)$ & $-0.12941(12)$ & $0.0746(6)$ \\
\hline $\mathrm{H} 26 \mathrm{~A}$ & 0.5062 & 0.9787 & -0.1011 & $0.090^{*}$ \\
\hline H26B & 0.4387 & 0.9693 & -0.1682 & $0.090^{*}$ \\
\hline $\mathrm{H} 26 \mathrm{C}$ & 0.4976 & 0.8790 & -0.1572 & $0.090^{*}$ \\
\hline $\mathrm{C} 27$ & $0.19236(14)$ & $0.8052(2)$ & $0.25240(14)$ & $0.1039(8)$ \\
\hline $\mathrm{H} 27 \mathrm{~A}$ & 0.2417 & 0.7812 & 0.2692 & $0.125^{*}$ \\
\hline $\mathrm{H} 27 \mathrm{~B}$ & 0.1736 & 0.7702 & 0.2030 & $0.125^{*}$ \\
\hline $\mathrm{H} 27 \mathrm{C}$ & 0.1613 & 0.7895 & 0.2941 & $0.125^{*}$ \\
\hline $\mathrm{C} 28$ & $0.01998(8)$ & $0.31643(13)$ & $0.13395(9)$ & $0.0427(4)$ \\
\hline $\mathrm{C} 29$ & $-0.00418(8)$ & $0.41658(14)$ & $0.12093(9)$ & 0.0493 (4) \\
\hline $\mathrm{C} 30$ & $-0.07104(9)$ & $0.43797(15)$ & $0.07641(11)$ & $0.0598(5)$ \\
\hline $\mathrm{H} 30$ & -0.0869 & 0.5072 & 0.0679 & $0.072 *$ \\
\hline $\mathrm{C} 31$ & $-0.11253(9)$ & $0.35791(17)$ & $0.04575(10)$ & $0.0615(5)$ \\
\hline $\mathrm{H} 31$ & -0.1572 & 0.3724 & 0.0148 & $0.074 *$ \\
\hline $\mathrm{C} 32$ & $-0.09160(8)$ & $0.25468(15)$ & $0.05827(10)$ & $0.0511(4)$ \\
\hline $\mathrm{C} 33$ & $-0.13472(9)$ & $0.17240(18)$ & $0.02490(11)$ & $0.0632(5)$ \\
\hline H33 & -0.1789 & 0.1872 & -0.0069 & $0.076^{*}$ \\
\hline $\mathrm{C} 34$ & $-0.11440(9)$ & $0.07300(18)$ & $0.03721(11)$ & $0.0642(5)$ \\
\hline $\mathrm{H} 34$ & -0.1436 & 0.0188 & 0.0134 & $0.077^{*}$ \\
\hline $\mathrm{C} 35$ & $-0.04946(9)$ & $0.05029(15)$ & $0.08581(11)$ & $0.0560(5)$ \\
\hline $\mathrm{C} 36$ & $-0.00565(8)$ & $0.12704(14)$ & $0.11868(9)$ & $0.0488(4)$ \\
\hline $\mathrm{H} 36$ & 0.0381 & 0.1102 & 0.1506 & $0.059^{*}$ \\
\hline $\mathrm{C} 37$ & $-0.02517(8)$ & $0.23192(13)$ & $0.10545(9)$ & $0.0453(4)$ \\
\hline $\mathrm{C} 38$ & $0.09465(8)$ & $0.29910(13)$ & $0.17421(9)$ & $0.0417(4)$ \\
\hline C39 & $0.15548(7)$ & $0.35787(12)$ & $0.14272(9)$ & $0.0392(4)$ \\
\hline $\mathrm{C} 40$ & $0.19892(8)$ & $0.42532(12)$ & $0.19288(9)$ & $0.0423(4)$ \\
\hline $\mathrm{C} 41$ & $0.19107(9)$ & $0.43916(14)$ & $0.27562(10)$ & $0.0545(5)$ \\
\hline $\mathrm{H} 41$ & 0.1553 & 0.4010 & 0.2990 & $0.065^{*}$ \\
\hline $\mathrm{C} 42$ & $0.23375(10)$ & $0.50569(15)$ & $0.32078(11)$ & $0.0647(5)$ \\
\hline $\mathrm{H} 42$ & 0.2282 & 0.5122 & 0.3758 & $0.078^{*}$ \\
\hline $\mathrm{C} 43$ & $0.28638(10)$ & $0.56567(15)$ & $0.28822(11)$ & $0.0635(5)$ \\
\hline $\mathrm{H} 43$ & 0.3157 & 0.6122 & 0.3211 & $0.076^{*}$ \\
\hline $\mathrm{C} 44$ & $0.29504(9)$ & $0.55677(14)$ & $0.20990(10)$ & $0.0559(5)$ \\
\hline $\mathrm{H} 44$ & 0.3303 & 0.5978 & 0.1881 & $0.067 *$ \\
\hline
\end{tabular}




$\begin{array}{lllll}\text { C45 } & 0.25198(8) & 0.48675(12) & 0.15987(9) & 0.0441(4) \\ \text { C46 } & 0.25969(8) & 0.47903(12) & 0.07897(9) & 0.0451(4) \\ \text { H46 } & 0.2939 & 0.5218 & 0.0571 & 0.054^{*} \\ \text { C47 } & 0.21865(8) & 0.41046(12) & 0.02949(9) & 0.0399(4) \\ \text { C48 } & 0.22752(8) & 0.40196(13) & -0.05310(9) & 0.0468(4) \\ \text { H48 } & 0.2602 & 0.4468 & -0.0756 & 0.056^{*} \\ \text { C49 } & 0.19020(8) & 0.33104(14) & -0.09977(9) & 0.0507(4) \\ \text { H49 } & 0.1968 & 0.3263 & -0.1546 & 0.061^{*} \\ \text { C50 } & 0.14141(8) & 0.26408(13) & -0.06721(10) & 0.0471(4) \\ \text { H50 } & 0.1167 & 0.2129 & -0.1000 & 0.057^{*} \\ \text { C51 } & 0.12934(8) & 0.27177(12) & 0.01032(9) & 0.0425(4) \\ \text { H51 } & 0.0956 & 0.2266 & 0.0307 & 0.051^{*} \\ \text { C52 } & 0.16631(7) & 0.34640(11) & 0.06179(9) & 0.0377(4) \\ \text { C53 } & 0.01975(11) & 0.59695(14) & 0.14541(13) & 0.0739(6) \\ \text { H53A } & -0.0258 & 0.6095 & 0.1680 & 0.089^{*} \\ \text { H53B } & 0.0135 & 0.6134 & 0.0884 & 0.089^{*} \\ \text { H53C } & 0.0579 & 0.6406 & 0.1725 & 0.089^{*} \\ \text { C54 } & 0.02583(11) & -0.08217(15) & 0.14738(13) & 0.0743(6) \\ \text { H54A } & 0.0692 & -0.0561 & 0.1259 & 0.089^{*} \\ \text { H54B } & 0.0282 & -0.1576 & 0.1509 & 0.089^{*} \\ \text { H54C } & 0.0232 & -0.0532 & 0.2006 & 0.089^{*}\end{array}$

Atomic displacement parameters $\left(\AA^{2}\right)$

\begin{tabular}{lllllll}
\hline & $U^{11}$ & $U^{22}$ & $U^{33}$ & $U^{12}$ & $U^{13}$ & $U^{23}$ \\
\hline O1 & $0.0497(7)$ & $0.0481(8)$ & $0.0986(10)$ & $-0.0031(6)$ & $0.0160(6)$ & $-0.0040(7)$ \\
O2 & $0.0689(8)$ & $0.0554(8)$ & $0.0714(8)$ & $0.0007(7)$ & $0.0113(7)$ & $0.0108(7)$ \\
O3 & $0.0797(10)$ & $0.1155(14)$ & $0.0815(10)$ & $0.0306(10)$ & $0.0133(7)$ & $-0.0171(10)$ \\
O4 & $0.0528(7)$ & $0.0567(8)$ & $0.0466(6)$ & $-0.0016(6)$ & $0.0000(5)$ & $0.0105(6)$ \\
O5 & $0.0507(7)$ & $0.0469(8)$ & $0.0862(9)$ & $0.0097(6)$ & $-0.0033(6)$ & $-0.0042(6)$ \\
O6 & $0.0615(8)$ & $0.0577(9)$ & $0.0931(10)$ & $-0.0130(7)$ & $0.0064(7)$ & $-0.0037(7)$ \\
C1 & $0.0421(9)$ & $0.0431(11)$ & $0.0622(11)$ & $0.0046(7)$ & $-0.0106(7)$ & $-0.0029(8)$ \\
C2 & $0.0519(10)$ & $0.0444(9)$ & $0.0644(11)$ & $-0.0027(8)$ & $-0.0145(8)$ & $0.0071(9)$ \\
C3 & $0.0748(13)$ & $0.0473(9)$ & $0.0867(14)$ & $0.0002(11)$ & $-0.0073(11)$ & $0.0039(11)$ \\
C4 & $0.0799(14)$ & $0.0458(12)$ & $0.0927(15)$ & $0.0021(11)$ & $-0.0176(11)$ & $0.0017(11)$ \\
C5 & $0.0575(11)$ & $0.0570(13)$ & $0.0759(13)$ & $0.0140(9)$ & $-0.0194(8)$ & $-0.0129(9)$ \\
C6 & $0.0759(14)$ & $0.0768(16)$ & $0.0895(16)$ & $0.0238(12)$ & $-0.0191(12)$ & $-0.0249(12)$ \\
C7 & $0.0665(13)$ & $0.0909(13)$ & $0.0795(15)$ & $0.0388(13)$ & $-0.0140(11)$ & $-0.0362(13)$ \\
C8 & $0.0559(11)$ & $0.0943(13)$ & $0.0690(12)$ & $0.0231(11)$ & $-0.0064(8)$ & $-0.0194(11)$ \\
C9 & $0.0476(10)$ & $0.0622(13)$ & $0.0636(11)$ & $0.0168(9)$ & $-0.0091(8)$ & $-0.0148(9)$ \\
C10 & $0.0451(9)$ & $0.0512(12)$ & $0.0673(11)$ & $0.0077(8)$ & $-0.0180(7)$ & $-0.0071(9)$ \\
C11 & $0.0436(9)$ & $0.0449(11)$ & $0.0547(10)$ & $-0.0011(8)$ & $-0.0016(8)$ & $0.0019(8)$ \\
C12 & $0.0440(9)$ & $0.0386(10)$ & $0.0511(9)$ & $0.0005(7)$ & $0.0045(7)$ & $0.0058(8)$ \\
C13 & $0.0540(10)$ & $0.0425(10)$ & $0.0458(9)$ & $-0.0050(8)$ & $0.0043(8)$ & $0.0057(8)$ \\
C14 & $0.0630(11)$ & $0.0551(12)$ & $0.0554(11)$ & $-0.0042(9)$ & $0.0006(9)$ & $0.0037(9)$ \\
C15 & $0.0831(14)$ & $0.0661(14)$ & $0.0499(11)$ & $-0.0146(12)$ & $-0.0027(10)$ & $0.0033(10)$ \\
C16 & $0.0927(16)$ & $0.0626(13)$ & $0.0500(11)$ & $-0.0108(12)$ & $0.0181(11)$ & $-0.0042(10)$ \\
C17 & $0.0749(13)$ & $0.0582(12)$ & $0.0546(11)$ & $-0.0043(10)$ & $0.0228(10)$ & $-0.0013(10)$ \\
& & & & & &
\end{tabular}




\begin{tabular}{|c|c|c|c|c|c|c|}
\hline $\mathrm{C} 18$ & $0.0569(10)$ & $0.0462(11)$ & $0.0480(10)$ & $-0.0024(8)$ & $0.0146(8)$ & $0.0037(8)$ \\
\hline C19 & $0.0497(10)$ & $0.0526(11)$ & $0.0602(11)$ & $0.0027(9)$ & $0.0138(8)$ & $0.0003(9)$ \\
\hline $\mathrm{C} 20$ & $0.0463(9)$ & $0.0455(10)$ & $0.0577(10)$ & $0.0012(8)$ & $0.0053(8)$ & $0.0022(9)$ \\
\hline $\mathrm{C} 21$ & $0.0479(10)$ & $0.0583(12)$ & $0.0771(13)$ & $0.0071(9)$ & $-0.0028(9)$ & $-0.0041(10)$ \\
\hline $\mathrm{C} 22$ & $0.0633(12)$ & $0.0682(14)$ & $0.0748(14)$ & $0.0114(11)$ & $-0.0169(10)$ & $-0.0125(11)$ \\
\hline $\mathrm{C} 23$ & $0.0733(13)$ & $0.0635(13)$ & $0.0603(11)$ & $0.0150(11)$ & $-0.0070(10)$ & $-0.0131(10)$ \\
\hline $\mathrm{C} 24$ & $0.0542(10)$ & $0.0513(11)$ & $0.0585(11)$ & $0.0115(9)$ & $0.0027(9)$ & $-0.0043(9)$ \\
\hline $\mathrm{C} 25$ & $0.0452(9)$ & $0.0378(10)$ & $0.0519(10)$ & $0.0019(7)$ & $0.0043(7)$ & $0.0023(8)$ \\
\hline $\mathrm{C} 26$ & $0.0636(12)$ & $0.0801(15)$ & $0.0802(14)$ & $-0.0126(11)$ & $0.0081(10)$ & $0.0194(12)$ \\
\hline $\mathrm{C} 27$ & $0.0995(19)$ & $0.131(3)$ & $0.0864(17)$ & $0.0015(18)$ & $0.0328(14)$ & $-0.0037(17)$ \\
\hline $\mathrm{C} 28$ & $0.0360(8)$ & $0.0505(11)$ & $0.0423(9)$ & $0.0027(8)$ & $0.0074(7)$ & $0.0028(8)$ \\
\hline $\mathrm{C} 29$ & $0.0413(9)$ & $0.0532(12)$ & $0.0538(10)$ & $0.0047(8)$ & $0.0066(8)$ & $0.0006(9)$ \\
\hline $\mathrm{C} 30$ & $0.0466(10)$ & $0.0613(13)$ & $0.0708(12)$ & $0.0145(9)$ & $0.0029(9)$ & $0.0057(10)$ \\
\hline $\mathrm{C} 31$ & $0.0378(9)$ & $0.0843(15)$ & $0.0610(11)$ & $0.0096(10)$ & $-0.0002(8)$ & $0.0039(11)$ \\
\hline $\mathrm{C} 32$ & $0.0354(8)$ & $0.0676(13)$ & $0.0507(10)$ & $0.0008(9)$ & $0.0070(7)$ & $0.0005(9)$ \\
\hline C33 & $0.0403(9)$ & $0.0884(16)$ & $0.0601(11)$ & $-0.0058(10)$ & $0.0023(8)$ & $-0.0046(11)$ \\
\hline $\mathrm{C} 34$ & $0.0465(10)$ & $0.0803(16)$ & $0.0659(12)$ & $-0.0181(10)$ & $0.0065(9)$ & $-0.0113(11)$ \\
\hline $\mathrm{C} 35$ & $0.0487(10)$ & $0.0583(13)$ & $0.0626(11)$ & $-0.0070(9)$ & $0.0135(8)$ & $-0.0020(9)$ \\
\hline $\mathrm{C} 36$ & $0.0403(9)$ & $0.0555(12)$ & $0.0513(10)$ & $-0.0040(8)$ & $0.0075(7)$ & $-0.0002(8)$ \\
\hline C37 & $0.0356(8)$ & $0.0575(12)$ & $0.0439(9)$ & $-0.0011(8)$ & $0.0090(7)$ & $0.0022(8)$ \\
\hline $\mathrm{C} 38$ & $0.0413(9)$ & $0.0434(10)$ & $0.0407(9)$ & $0.0030(7)$ & $0.0054(7)$ & $-0.0006(8)$ \\
\hline C39 & $0.0337(7)$ & $0.0410(9)$ & $0.0424(8)$ & $0.0061(7)$ & $0.0021(6)$ & $0.0020(7)$ \\
\hline $\mathrm{C} 40$ & $0.0361(8)$ & $0.0475(10)$ & $0.0427(9)$ & $0.0067(7)$ & $0.0016(7)$ & $0.0000(8)$ \\
\hline $\mathrm{C} 41$ & $0.0502(10)$ & $0.0685(13)$ & $0.0443(9)$ & $0.0011(9)$ & $0.0031(8)$ & $-0.0048(9)$ \\
\hline $\mathrm{C} 42$ & $0.0596(11)$ & $0.0867(15)$ & $0.0463(10)$ & $0.0019(11)$ & $-0.0004(9)$ & $-0.0124(10)$ \\
\hline $\mathrm{C} 43$ & $0.0547(11)$ & $0.0728(13)$ & $0.0599(12)$ & $-0.0031(10)$ & $-0.0076(9)$ & $-0.0160(10)$ \\
\hline $\mathrm{C} 44$ & $0.0461(9)$ & $0.0594(12)$ & $0.0603(11)$ & $-0.0049(9)$ & $-0.0031(8)$ & $-0.0075(9)$ \\
\hline $\mathrm{C} 45$ & $0.0377(8)$ & $0.0462(10)$ & $0.0472(9)$ & $0.0024(7)$ & $-0.0010(7)$ & $-0.0028(8)$ \\
\hline $\mathrm{C} 46$ & $0.0368(8)$ & $0.0458(10)$ & $0.0530(10)$ & $-0.0016(7)$ & $0.0059(7)$ & $0.0017(8)$ \\
\hline $\mathrm{C} 47$ & $0.0341(8)$ & $0.0408(9)$ & $0.0444(9)$ & $0.0052(7)$ & $0.0031(7)$ & $0.0005(7)$ \\
\hline $\mathrm{C} 48$ & $0.0396(8)$ & $0.0560(11)$ & $0.0458(9)$ & $-0.0012(8)$ & $0.0085(7)$ & $0.0041(8)$ \\
\hline C49 & $0.0443(9)$ & $0.0664(12)$ & $0.0422(9)$ & $-0.0018(9)$ & $0.0075(7)$ & $-0.0054(9)$ \\
\hline $\mathrm{C} 50$ & $0.0399(8)$ & $0.0532(11)$ & $0.0483(10)$ & $-0.0018(8)$ & $0.0057(7)$ & $-0.0097(8)$ \\
\hline $\mathrm{C} 51$ & $0.0345(8)$ & $0.0442(10)$ & $0.0486(9)$ & $0.0010(7)$ & $0.0045(7)$ & $-0.0012(8)$ \\
\hline $\mathrm{C} 52$ & $0.0323(7)$ & $0.0382(9)$ & $0.0424(9)$ & $0.0055(7)$ & $0.0026(6)$ & $0.0015(7)$ \\
\hline C53 & $0.0721(13)$ & $0.0509(13)$ & $0.0974(15)$ & $0.0119(10)$ & $0.0038(11)$ & $0.0021(11)$ \\
\hline C54 & $0.0737(14)$ & $0.0528(13)$ & $0.0964(15)$ & $-0.0027(11)$ & $0.0094(12)$ & $0.0021(11)$ \\
\hline
\end{tabular}

Geometric parameters $\left(A,{ }^{\circ}\right)$

\begin{tabular}{llll}
\hline $\mathrm{O} 1-\mathrm{C} 11$ & $1.2226(18)$ & $\mathrm{C} 26-\mathrm{H} 26 \mathrm{~B}$ & 0.9800 \\
$\mathrm{O} 2-\mathrm{C} 2$ & $1.339(2)$ & $\mathrm{C} 26-\mathrm{H} 26 \mathrm{C}$ & 0.9800 \\
$\mathrm{O} 2-\mathrm{C} 26$ & $1.432(2)$ & $\mathrm{C} 27-\mathrm{H} 27 \mathrm{~A}$ & 0.9800 \\
$\mathrm{O} 3-\mathrm{C} 8$ & $1.364(2)$ & $\mathrm{C} 27-\mathrm{H} 27 \mathrm{~B}$ & 0.9800 \\
$\mathrm{O} 3-\mathrm{C} 27$ & $1.423(3)$ & $\mathrm{C} 27-\mathrm{H} 27 \mathrm{C}$ & 0.9800 \\
$\mathrm{O} 4-\mathrm{C} 38$ & $1.2203(17)$ & $\mathrm{C} 28-\mathrm{C} 29$ & $1.383(2)$ \\
$\mathrm{O} 5-\mathrm{C} 29$ & $1.3574(19)$ & $\mathrm{C} 28-\mathrm{C} 37$ & $1.431(2)$ \\
$\mathrm{O} 5-\mathrm{C} 53$ & $1.425(2)$ & $\mathrm{C} 28-\mathrm{C} 38$ & $1.494(2)$
\end{tabular}




\begin{tabular}{|c|c|c|c|}
\hline $\mathrm{O} 6-\mathrm{C} 35$ & $1.365(2)$ & $\mathrm{C} 29-\mathrm{C} 30$ & $1.407(2)$ \\
\hline $\mathrm{O} 6-\mathrm{C} 54$ & $1.417(2)$ & $\mathrm{C} 30-\mathrm{C} 31$ & $1.361(3)$ \\
\hline $\mathrm{C} 1-\mathrm{C} 2$ & $1.409(2)$ & $\mathrm{C} 30-\mathrm{H} 30$ & 0.9500 \\
\hline $\mathrm{C} 1-\mathrm{C} 10$ & $1.424(2)$ & $\mathrm{C} 31-\mathrm{C} 32$ & $1.403(2)$ \\
\hline $\mathrm{C} 1-\mathrm{C} 11$ & $1.487(2)$ & C $31-\mathrm{H} 31$ & 0.9500 \\
\hline $\mathrm{C} 2-\mathrm{C} 3$ & $1.413(3)$ & $\mathrm{C} 32-\mathrm{C} 33$ & $1.414(2)$ \\
\hline $\mathrm{C} 3-\mathrm{C} 4$ & $1.343(3)$ & $\mathrm{C} 32-\mathrm{C} 37$ & $1.425(2)$ \\
\hline $\mathrm{C} 3-\mathrm{H} 3$ & 0.9500 & $\mathrm{C} 33-\mathrm{C} 34$ & $1.352(3)$ \\
\hline $\mathrm{C} 4-\mathrm{C} 5$ & $1.402(3)$ & C33-H33 & 0.9500 \\
\hline $\mathrm{C} 4-\mathrm{H} 4$ & 0.9500 & $\mathrm{C} 34-\mathrm{C} 35$ & $1.414(2)$ \\
\hline $\mathrm{C} 5-\mathrm{C} 6$ & $1.390(3)$ & C34-H34 & 0.9500 \\
\hline $\mathrm{C} 5-\mathrm{C} 10$ & $1.444(3)$ & $\mathrm{C} 35-\mathrm{C} 36$ & $1.364(2)$ \\
\hline $\mathrm{C} 6-\mathrm{C} 7$ & $1.350(3)$ & $\mathrm{C} 36-\mathrm{C} 37$ & $1.418(2)$ \\
\hline C6- 6 - 6 & 0.9500 & $\mathrm{C} 36-\mathrm{H} 36$ & 0.9500 \\
\hline $\mathrm{C} 7-\mathrm{C} 8$ & $1.429(3)$ & $\mathrm{C} 38-\mathrm{C} 39$ & $1.509(2)$ \\
\hline $\mathrm{C} 7-\mathrm{H} 7$ & 0.9500 & $\mathrm{C} 39-\mathrm{C} 40$ & $1.408(2)$ \\
\hline $\mathrm{C} 8-\mathrm{C} 9$ & $1.359(2)$ & C $39-\mathrm{C} 52$ & $1.4113(19)$ \\
\hline $\mathrm{C} 9-\mathrm{C} 10$ & $1.416(2)$ & $\mathrm{C} 40-\mathrm{C} 45$ & $1.428(2)$ \\
\hline $\mathrm{C} 9-\mathrm{H} 9$ & 0.9500 & $\mathrm{C} 40-\mathrm{C} 41$ & $1.432(2)$ \\
\hline $\mathrm{C} 11-\mathrm{C} 12$ & $1.501(2)$ & $\mathrm{C} 41-\mathrm{C} 42$ & $1.350(2)$ \\
\hline $\mathrm{C} 12-\mathrm{C} 25$ & $1.402(2)$ & $\mathrm{C} 41-\mathrm{H} 41$ & 0.9500 \\
\hline $\mathrm{C} 12-\mathrm{C} 13$ & 1.407 (2) & $\mathrm{C} 42-\mathrm{C} 43$ & 1.409 (3) \\
\hline $\mathrm{C} 13-\mathrm{C} 14$ & $1.431(2)$ & $\mathrm{C} 42-\mathrm{H} 42$ & 0.9500 \\
\hline $\mathrm{C} 13-\mathrm{C} 18$ & $1.432(2)$ & $\mathrm{C} 43-\mathrm{C} 44$ & $1.355(2)$ \\
\hline $\mathrm{C} 14-\mathrm{C} 15$ & $1.365(2)$ & $\mathrm{C} 43-\mathrm{H} 43$ & 0.9500 \\
\hline C14-H14 & 0.9500 & $\mathrm{C} 44-\mathrm{C} 45$ & $1.424(2)$ \\
\hline $\mathrm{C} 15-\mathrm{C} 16$ & $1.407(3)$ & $\mathrm{C} 44-\mathrm{H} 44$ & 0.9500 \\
\hline $\mathrm{C} 15-\mathrm{H} 15$ & 0.9500 & $\mathrm{C} 45-\mathrm{C} 46$ & $1.393(2)$ \\
\hline $\mathrm{C} 16-\mathrm{C} 17$ & $1.342(3)$ & $\mathrm{C} 46-\mathrm{C} 47$ & $1.389(2)$ \\
\hline $\mathrm{C} 16-\mathrm{H} 16$ & 0.9500 & $\mathrm{C} 46-\mathrm{H} 46$ & 0.9500 \\
\hline $\mathrm{C} 17-\mathrm{C} 18$ & $1.433(2)$ & $\mathrm{C} 47-\mathrm{C} 48$ & $1.427(2)$ \\
\hline $\mathrm{C} 17-\mathrm{H} 17$ & 0.9500 & $\mathrm{C} 47-\mathrm{C} 52$ & $1.433(2)$ \\
\hline $\mathrm{C} 18-\mathrm{C} 19$ & $1.385(2)$ & $\mathrm{C} 48-\mathrm{C} 49$ & $1.351(2)$ \\
\hline $\mathrm{C} 19-\mathrm{C} 20$ & $1.386(2)$ & $\mathrm{C} 48-\mathrm{H} 48$ & 0.9500 \\
\hline C19-H19 & 0.9500 & $\mathrm{C} 49-\mathrm{C} 50$ & $1.410(2)$ \\
\hline $\mathrm{C} 20-\mathrm{C} 21$ & $1.426(2)$ & $\mathrm{C} 49-\mathrm{H} 49$ & 0.9500 \\
\hline $\mathrm{C} 20-\mathrm{C} 25$ & $1.435(2)$ & $\mathrm{C} 50-\mathrm{C} 51$ & $1.357(2)$ \\
\hline $\mathrm{C} 21-\mathrm{C} 22$ & $1.344(2)$ & $\mathrm{C} 50-\mathrm{H} 50$ & 0.9500 \\
\hline $\mathrm{C} 21-\mathrm{H} 21$ & 0.9500 & $\mathrm{C} 51-\mathrm{C} 52$ & $1.425(2)$ \\
\hline $\mathrm{C} 22-\mathrm{C} 23$ & $1.408(3)$ & C51-H51 & 0.9500 \\
\hline $\mathrm{C} 22-\mathrm{H} 22$ & 0.9500 & $\mathrm{C} 53-\mathrm{H} 53 \mathrm{~A}$ & 0.9800 \\
\hline $\mathrm{C} 23-\mathrm{C} 24$ & $1.356(2)$ & С53-H53B & 0.9800 \\
\hline $\mathrm{C} 23-\mathrm{H} 23$ & 0.9500 & $\mathrm{C} 53-\mathrm{H} 53 \mathrm{C}$ & 0.9800 \\
\hline $\mathrm{C} 24-\mathrm{C} 25$ & $1.425(2)$ & $\mathrm{C} 54-\mathrm{H} 54 \mathrm{~A}$ & 0.9800 \\
\hline $\mathrm{C} 24-\mathrm{H} 24$ & 0.9500 & С54-H54B & 0.9800 \\
\hline $\mathrm{C} 26-\mathrm{H} 26 \mathrm{~A}$ & 0.9800 & C54-H54C & 0.9800 \\
\hline $\mathrm{C} 2-\mathrm{O} 2-\mathrm{C} 26$ & $119.82(15)$ & $\mathrm{H} 27 \mathrm{~A}-\mathrm{C} 27-\mathrm{H} 27 \mathrm{C}$ & 109.5 \\
\hline
\end{tabular}




\begin{tabular}{|c|c|c|c|}
\hline $\mathrm{C} 8-\mathrm{O} 3-\mathrm{C} 27$ & $118.70(17)$ & $\mathrm{H} 27 \mathrm{~B}-\mathrm{C} 27-\mathrm{H} 27 \mathrm{C}$ & 109.5 \\
\hline $\mathrm{C} 29-\mathrm{O} 5-\mathrm{C} 53$ & $119.77(14)$ & $\mathrm{C} 29-\mathrm{C} 28-\mathrm{C} 37$ & $119.79(14)$ \\
\hline $\mathrm{C} 35-\mathrm{O} 6-\mathrm{C} 54$ & $117.68(14)$ & $\mathrm{C} 29-\mathrm{C} 28-\mathrm{C} 38$ & $118.86(15)$ \\
\hline $\mathrm{C} 2-\mathrm{C} 1-\mathrm{C} 10$ & $120.40(16)$ & $\mathrm{C} 37-\mathrm{C} 28-\mathrm{C} 38$ & $121.28(14)$ \\
\hline $\mathrm{C} 2-\mathrm{C} 1-\mathrm{C} 11$ & $119.08(16)$ & $\mathrm{O} 5-\mathrm{C} 29-\mathrm{C} 28$ & $115.55(14)$ \\
\hline $\mathrm{C} 10-\mathrm{C} 1-\mathrm{C} 11$ & $120.47(16)$ & $\mathrm{O} 5-\mathrm{C} 29-\mathrm{C} 30$ & $122.95(16)$ \\
\hline $\mathrm{O} 2-\mathrm{C} 2-\mathrm{C} 1$ & $117.57(15)$ & $\mathrm{C} 28-\mathrm{C} 29-\mathrm{C} 30$ & $121.47(17)$ \\
\hline $\mathrm{O} 2-\mathrm{C} 2-\mathrm{C} 3$ & $122.18(17)$ & $\mathrm{C} 31-\mathrm{C} 30-\mathrm{C} 29$ & $118.89(17)$ \\
\hline $\mathrm{C} 1-\mathrm{C} 2-\mathrm{C} 3$ & $120.15(18)$ & $\mathrm{C} 31-\mathrm{C} 30-\mathrm{H} 30$ & 120.6 \\
\hline $\mathrm{C} 4-\mathrm{C} 3-\mathrm{C} 2$ & $119.4(2)$ & $\mathrm{C} 29-\mathrm{C} 30-\mathrm{H} 30$ & 120.6 \\
\hline $\mathrm{C} 4-\mathrm{C} 3-\mathrm{H} 3$ & 120.3 & $\mathrm{C} 30-\mathrm{C} 31-\mathrm{C} 32$ & $122.25(16)$ \\
\hline $\mathrm{C} 2-\mathrm{C} 3-\mathrm{H} 3$ & 120.3 & $\mathrm{C} 30-\mathrm{C} 31-\mathrm{H} 31$ & 118.9 \\
\hline $\mathrm{C} 3-\mathrm{C} 4-\mathrm{C} 5$ & $123.7(2)$ & $\mathrm{C} 32-\mathrm{C} 31-\mathrm{H} 31$ & 118.9 \\
\hline $\mathrm{C} 3-\mathrm{C} 4-\mathrm{H} 4$ & 118.2 & $\mathrm{C} 31-\mathrm{C} 32-\mathrm{C} 33$ & $121.58(16)$ \\
\hline $\mathrm{C} 5-\mathrm{C} 4-\mathrm{H} 4$ & 118.2 & $\mathrm{C} 31-\mathrm{C} 32-\mathrm{C} 37$ & $119.39(16)$ \\
\hline $\mathrm{C} 6-\mathrm{C} 5-\mathrm{C} 4$ & $123.1(2)$ & $\mathrm{C} 33-\mathrm{C} 32-\mathrm{C} 37$ & $119.03(17)$ \\
\hline $\mathrm{C} 6-\mathrm{C} 5-\mathrm{C} 10$ & $118.4(2)$ & $\mathrm{C} 34-\mathrm{C} 33-\mathrm{C} 32$ & $121.39(17)$ \\
\hline $\mathrm{C} 4-\mathrm{C} 5-\mathrm{C} 10$ & $118.46(18)$ & $\mathrm{C} 34-\mathrm{C} 33-\mathrm{H} 33$ & 119.3 \\
\hline $\mathrm{C} 7-\mathrm{C} 6-\mathrm{C} 5$ & $123.4(2)$ & $\mathrm{C} 32-\mathrm{C} 33-\mathrm{H} 33$ & 119.3 \\
\hline $\mathrm{C} 7-\mathrm{C} 6-\mathrm{H} 6$ & 118.3 & $\mathrm{C} 33-\mathrm{C} 34-\mathrm{C} 35$ & $119.60(18)$ \\
\hline $\mathrm{C} 5-\mathrm{C} 6-\mathrm{H} 6$ & 118.3 & $\mathrm{C} 33-\mathrm{C} 34-\mathrm{H} 34$ & 120.2 \\
\hline $\mathrm{C} 6-\mathrm{C} 7-\mathrm{C} 8$ & $117.9(2)$ & $\mathrm{C} 35-\mathrm{C} 34-\mathrm{H} 34$ & 120.2 \\
\hline $\mathrm{C} 6-\mathrm{C} 7-\mathrm{H} 7$ & 121.0 & $\mathrm{C} 36-\mathrm{C} 35-\mathrm{O} 6$ & $124.78(16)$ \\
\hline $\mathrm{C} 8-\mathrm{C} 7-\mathrm{H} 7$ & 121.0 & $\mathrm{C} 36-\mathrm{C} 35-\mathrm{C} 34$ & $121.17(18)$ \\
\hline $\mathrm{C} 9-\mathrm{C} 8-\mathrm{O} 3$ & $124.8(2)$ & $\mathrm{O} 6-\mathrm{C} 35-\mathrm{C} 34$ & $114.05(17)$ \\
\hline $\mathrm{C} 9-\mathrm{C} 8-\mathrm{C} 7$ & $121.7(2)$ & $\mathrm{C} 35-\mathrm{C} 36-\mathrm{C} 37$ & $120.26(16)$ \\
\hline $\mathrm{O} 3-\mathrm{C} 8-\mathrm{C} 7$ & $113.5(2)$ & $\mathrm{C} 35-\mathrm{C} 36-\mathrm{H} 36$ & 119.9 \\
\hline $\mathrm{C} 8-\mathrm{C} 9-\mathrm{C} 10$ & $120.29(19)$ & $\mathrm{C} 37-\mathrm{C} 36-\mathrm{H} 36$ & 119.9 \\
\hline $\mathrm{C} 8-\mathrm{C} 9-\mathrm{H} 9$ & 119.9 & $\mathrm{C} 36-\mathrm{C} 37-\mathrm{C} 32$ & $118.52(15)$ \\
\hline $\mathrm{C} 10-\mathrm{C} 9-\mathrm{H} 9$ & 119.9 & $\mathrm{C} 36-\mathrm{C} 37-\mathrm{C} 28$ & $123.39(14)$ \\
\hline $\mathrm{C} 9-\mathrm{C} 10-\mathrm{C} 1$ & $123.81(17)$ & $\mathrm{C} 32-\mathrm{C} 37-\mathrm{C} 28$ & $118.02(15)$ \\
\hline $\mathrm{C} 9-\mathrm{C} 10-\mathrm{C} 5$ & $118.23(17)$ & $\mathrm{O} 4-\mathrm{C} 38-\mathrm{C} 28$ & $121.83(14)$ \\
\hline $\mathrm{C} 1-\mathrm{C} 10-\mathrm{C} 5$ & $117.93(17)$ & $\mathrm{O} 4-\mathrm{C} 38-\mathrm{C} 39$ & $120.82(13)$ \\
\hline $\mathrm{O} 1-\mathrm{C} 11-\mathrm{C} 1$ & $121.49(15)$ & $\mathrm{C} 28-\mathrm{C} 38-\mathrm{C} 39$ & $117.33(13)$ \\
\hline $\mathrm{O} 1-\mathrm{C} 11-\mathrm{C} 12$ & $119.62(15)$ & $\mathrm{C} 40-\mathrm{C} 39-\mathrm{C} 52$ & $120.94(13)$ \\
\hline $\mathrm{C} 1-\mathrm{C} 11-\mathrm{C} 12$ & $118.89(14)$ & $\mathrm{C} 40-\mathrm{C} 39-\mathrm{C} 38$ & $120.26(13)$ \\
\hline $\mathrm{C} 25-\mathrm{C} 12-\mathrm{C} 13$ & $120.90(14)$ & $\mathrm{C} 52-\mathrm{C} 39-\mathrm{C} 38$ & $118.78(13)$ \\
\hline $\mathrm{C} 25-\mathrm{C} 12-\mathrm{C} 11$ & $119.13(14)$ & $\mathrm{C} 39-\mathrm{C} 40-\mathrm{C} 45$ & $119.09(14)$ \\
\hline $\mathrm{C} 13-\mathrm{C} 12-\mathrm{C} 11$ & $119.97(14)$ & $\mathrm{C} 39-\mathrm{C} 40-\mathrm{C} 41$ & $123.44(14)$ \\
\hline $\mathrm{C} 12-\mathrm{C} 13-\mathrm{C} 14$ & $123.29(15)$ & $\mathrm{C} 45-\mathrm{C} 40-\mathrm{C} 41$ & $117.42(14)$ \\
\hline $\mathrm{C} 12-\mathrm{C} 13-\mathrm{C} 18$ & $118.84(15)$ & $\mathrm{C} 42-\mathrm{C} 41-\mathrm{C} 40$ & $120.96(17)$ \\
\hline $\mathrm{C} 14-\mathrm{C} 13-\mathrm{C} 18$ & $117.85(15)$ & $\mathrm{C} 42-\mathrm{C} 41-\mathrm{H} 41$ & 119.5 \\
\hline $\mathrm{C} 15-\mathrm{C} 14-\mathrm{C} 13$ & $120.70(18)$ & $\mathrm{C} 40-\mathrm{C} 41-\mathrm{H} 41$ & 119.5 \\
\hline $\mathrm{C} 15-\mathrm{C} 14-\mathrm{H} 14$ & 119.6 & $\mathrm{C} 41-\mathrm{C} 42-\mathrm{C} 43$ & $121.50(17)$ \\
\hline $\mathrm{C} 13-\mathrm{C} 14-\mathrm{H} 14$ & 119.6 & $\mathrm{C} 41-\mathrm{C} 42-\mathrm{H} 42$ & 119.3 \\
\hline $\mathrm{C} 14-\mathrm{C} 15-\mathrm{C} 16$ & $120.85(18)$ & $\mathrm{C} 43-\mathrm{C} 42-\mathrm{H} 42$ & 119.3 \\
\hline $\mathrm{C} 14-\mathrm{C} 15-\mathrm{H} 15$ & 119.6 & $\mathrm{C} 44-\mathrm{C} 43-\mathrm{C} 42$ & $119.81(17)$ \\
\hline $\mathrm{C} 16-\mathrm{C} 15-\mathrm{H} 15$ & 119.6 & $\mathrm{C} 44-\mathrm{C} 43-\mathrm{H} 43$ & 120.1 \\
\hline
\end{tabular}


$\mathrm{C} 17-\mathrm{C} 16-\mathrm{C} 15$

$\mathrm{C} 17-\mathrm{C} 16-\mathrm{H} 16$

$\mathrm{C} 15-\mathrm{C} 16-\mathrm{H} 16$

$\mathrm{C} 16-\mathrm{C} 17-\mathrm{C} 18$

$\mathrm{C} 16-\mathrm{C} 17-\mathrm{H} 17$

$\mathrm{C} 18-\mathrm{C} 17-\mathrm{H} 17$

C19-C18-C13

C19-C18-C17

$\mathrm{C} 13-\mathrm{C} 18-\mathrm{C} 17$

$\mathrm{C} 18-\mathrm{C} 19-\mathrm{C} 20$

C18-C19-H19

$\mathrm{C} 20-\mathrm{C} 19-\mathrm{H} 19$

$\mathrm{C} 19-\mathrm{C} 20-\mathrm{C} 21$

$\mathrm{C} 19-\mathrm{C} 20-\mathrm{C} 25$

$\mathrm{C} 21-\mathrm{C} 20-\mathrm{C} 25$

$\mathrm{C} 22-\mathrm{C} 21-\mathrm{C} 20$

$\mathrm{C} 22-\mathrm{C} 21-\mathrm{H} 21$

$\mathrm{C} 20-\mathrm{C} 21-\mathrm{H} 21$

$\mathrm{C} 21-\mathrm{C} 22-\mathrm{C} 23$

$\mathrm{C} 21-\mathrm{C} 22-\mathrm{H} 22$

$\mathrm{C} 23-\mathrm{C} 22-\mathrm{H} 22$

$\mathrm{C} 24-\mathrm{C} 23-\mathrm{C} 22$

$\mathrm{C} 24-\mathrm{C} 23-\mathrm{H} 23$

$\mathrm{C} 22-\mathrm{C} 23-\mathrm{H} 23$

$\mathrm{C} 23-\mathrm{C} 24-\mathrm{C} 25$

$\mathrm{C} 23-\mathrm{C} 24-\mathrm{H} 24$

$\mathrm{C} 25-\mathrm{C} 24-\mathrm{H} 24$

$\mathrm{C} 12-\mathrm{C} 25-\mathrm{C} 24$

$\mathrm{C} 12-\mathrm{C} 25-\mathrm{C} 20$

$\mathrm{C} 24-\mathrm{C} 25-\mathrm{C} 20$

$\mathrm{O} 2-\mathrm{C} 26-\mathrm{H} 26 \mathrm{~A}$

$\mathrm{O} 2-\mathrm{C} 26-\mathrm{H} 26 \mathrm{~B}$

$\mathrm{H} 26 \mathrm{~A}-\mathrm{C} 26-\mathrm{H} 26 \mathrm{~B}$

$\mathrm{O} 2-\mathrm{C} 26-\mathrm{H} 26 \mathrm{C}$

$\mathrm{H} 26 \mathrm{~A}-\mathrm{C} 26-\mathrm{H} 26 \mathrm{C}$

$\mathrm{H} 26 \mathrm{~B}-\mathrm{C} 26-\mathrm{H} 26 \mathrm{C}$

$\mathrm{O} 3-\mathrm{C} 27-\mathrm{H} 27 \mathrm{~A}$

$\mathrm{O} 3-\mathrm{C} 27-\mathrm{H} 27 \mathrm{~B}$

$\mathrm{H} 27 \mathrm{~A}-\mathrm{C} 27-\mathrm{H} 27 \mathrm{~B}$

$\mathrm{O} 3-\mathrm{C} 27-\mathrm{H} 27 \mathrm{C}$

$\mathrm{C} 26-\mathrm{O} 2-\mathrm{C} 2-\mathrm{C} 1$

$\mathrm{C} 26-\mathrm{O} 2-\mathrm{C} 2-\mathrm{C} 3$

$\mathrm{C} 10-\mathrm{C} 1-\mathrm{C} 2-\mathrm{O} 2$

$\mathrm{C} 11-\mathrm{C} 1-\mathrm{C} 2-\mathrm{O} 2$

$\mathrm{C} 10-\mathrm{C} 1-\mathrm{C} 2-\mathrm{C} 3$

$\mathrm{C} 11-\mathrm{C} 1-\mathrm{C} 2-\mathrm{C} 3$

$\mathrm{O} 2-\mathrm{C} 2-\mathrm{C} 3-\mathrm{C} 4$
120.71 (18)

119.6

119.6

121.03 (19)

119.5

119.5

119.55 (15)

121.62 (16)

118.80 (16)

122.03 (16)

119.0

119.0

122.07 (16)

$119.11(15)$

118.80 (16)

121.47 (17)

119.3

119.3

119.97 (18)

120.0

120.0

120.98 (18)

119.5

119.5

121.32 (16)

119.3

119.3

123.32 (14)

119.25 (15)

117.36 (14)

109.5

109.5

109.5

109.5

109.5

109.5

109.5

109.5

109.5

109.5

-178.71 (14)

4.7 (2)

-176.99 (14)

0.7 (2)

-0.3 (2)

177.33 (15)

177.20 (17)
$\mathrm{C} 42-\mathrm{C} 43-\mathrm{H} 43$

$\mathrm{C} 43-\mathrm{C} 44-\mathrm{C} 45$

$\mathrm{C} 43-\mathrm{C} 44-\mathrm{H} 44$

$\mathrm{C} 45-\mathrm{C} 44-\mathrm{H} 44$

$\mathrm{C} 46-\mathrm{C} 45-\mathrm{C} 44$

$\mathrm{C} 46-\mathrm{C} 45-\mathrm{C} 40$

$\mathrm{C} 44-\mathrm{C} 45-\mathrm{C} 40$

$\mathrm{C} 47-\mathrm{C} 46-\mathrm{C} 45$

$\mathrm{C} 47-\mathrm{C} 46-\mathrm{H} 46$

$\mathrm{C} 45-\mathrm{C} 46-\mathrm{H} 46$

$\mathrm{C} 46-\mathrm{C} 47-\mathrm{C} 48$

$\mathrm{C} 46-\mathrm{C} 47-\mathrm{C} 52$

$\mathrm{C} 48-\mathrm{C} 47-\mathrm{C} 52$

$\mathrm{C} 49-\mathrm{C} 48-\mathrm{C} 47$

$\mathrm{C} 49-\mathrm{C} 48-\mathrm{H} 48$

$\mathrm{C} 47-\mathrm{C} 48-\mathrm{H} 48$

$\mathrm{C} 48-\mathrm{C} 49-\mathrm{C} 50$

$\mathrm{C} 48-\mathrm{C} 49-\mathrm{H} 49$

$\mathrm{C} 50-\mathrm{C} 49-\mathrm{H} 49$

$\mathrm{C} 51-\mathrm{C} 50-\mathrm{C} 49$

$\mathrm{C} 51-\mathrm{C} 50-\mathrm{H} 50$

$\mathrm{C} 49-\mathrm{C} 50-\mathrm{H} 50$

$\mathrm{C} 50-\mathrm{C} 51-\mathrm{C} 52$

$\mathrm{C} 50-\mathrm{C} 51-\mathrm{H} 51$

C52-C51-H51

C39-C52-C51

$\mathrm{C} 39-\mathrm{C} 52-\mathrm{C} 47$

$\mathrm{C} 51-\mathrm{C} 52-\mathrm{C} 47$

$\mathrm{O} 5-\mathrm{C} 53-\mathrm{H} 53 \mathrm{~A}$

$\mathrm{O} 5-\mathrm{C} 53-\mathrm{H} 53 \mathrm{~B}$

$\mathrm{H} 53 \mathrm{~A}-\mathrm{C} 53-\mathrm{H} 53 \mathrm{~B}$

O5-C53-H53C

$\mathrm{H} 53 \mathrm{~A}-\mathrm{C} 53-\mathrm{H} 53 \mathrm{C}$

$\mathrm{H} 53 \mathrm{~B}-\mathrm{C} 53-\mathrm{H} 53 \mathrm{C}$

$\mathrm{O} 6-\mathrm{C} 54-\mathrm{H} 54 \mathrm{~A}$

O6- C54-H54B

H54A-C54-H54B

O6- $554-\mathrm{H} 54 \mathrm{C}$

$\mathrm{H} 54 \mathrm{~A}-\mathrm{C} 54-\mathrm{H} 54 \mathrm{C}$

$\mathrm{H} 54 \mathrm{~B}-\mathrm{C} 54-\mathrm{H} 54 \mathrm{C}$

$\mathrm{C} 53-\mathrm{O} 5-\mathrm{C} 29-\mathrm{C} 28$

$\mathrm{C} 53-\mathrm{O} 5-\mathrm{C} 29-\mathrm{C} 30$

$\mathrm{C} 37-\mathrm{C} 28-\mathrm{C} 29-\mathrm{O} 5$

$\mathrm{C} 38-\mathrm{C} 28-\mathrm{C} 29-\mathrm{O} 5$

$\mathrm{C} 37-\mathrm{C} 28-\mathrm{C} 29-\mathrm{C} 30$

$\mathrm{C} 38-\mathrm{C} 28-\mathrm{C} 29-\mathrm{C} 30$

$\mathrm{O} 5-\mathrm{C} 29-\mathrm{C} 30-\mathrm{C} 31$
120.1

120.90 (17)

119.5

119.5

121.03 (15)

119.57 (14)

$119.39(15)$

$121.78(14)$

119.1

119.1

$121.48(14)$

$119.53(14)$

118.99 (14)

$121.00(15)$

119.5

119.5

120.14 (15)

119.9

119.9

120.90 (15)

119.6

119.6

121.23 (14)

119.4

119.4

123.45 (13)

118.93 (13)

117.59 (13)

109.5

109.5

109.5

109.5

109.5

109.5

109.5

109.5

109.5

109.5

109.5

109.5

179.33 (15)

-2.8 (2)

174.52 (13)

-8.5 (2)

-3.4 (2)

173.60 (14)

-177.69 (16) 


\begin{tabular}{|c|c|c|c|}
\hline $\mathrm{C} 1-\mathrm{C} 2-\mathrm{C} 3-\mathrm{C} 4$ & $0.7(3)$ & $\mathrm{C} 28-\mathrm{C} 29-\mathrm{C} 30-\mathrm{C} 31$ & $0.1(3)$ \\
\hline $\mathrm{C} 2-\mathrm{C} 3-\mathrm{C} 4-\mathrm{C} 5$ & $0.0(3)$ & $\mathrm{C} 29-\mathrm{C} 30-\mathrm{C} 31-\mathrm{C} 32$ & $1.3(3)$ \\
\hline $\mathrm{C} 3-\mathrm{C} 4-\mathrm{C} 5-\mathrm{C} 6$ & $179.76(19)$ & $\mathrm{C} 30-\mathrm{C} 31-\mathrm{C} 32-\mathrm{C} 33$ & $-178.92(16)$ \\
\hline $\mathrm{C} 3-\mathrm{C} 4-\mathrm{C} 5-\mathrm{C} 10$ & $-1.0(3)$ & $\mathrm{C} 30-\mathrm{C} 31-\mathrm{C} 32-\mathrm{C} 37$ & $0.8(3)$ \\
\hline $\mathrm{C} 4-\mathrm{C} 5-\mathrm{C} 6-\mathrm{C} 7$ & $-179.99(19)$ & $\mathrm{C} 31-\mathrm{C} 32-\mathrm{C} 33-\mathrm{C} 34$ & $-179.90(16)$ \\
\hline $\mathrm{C} 10-\mathrm{C} 5-\mathrm{C} 6-\mathrm{C} 7$ & $0.7(3)$ & $\mathrm{C} 37-\mathrm{C} 32-\mathrm{C} 33-\mathrm{C} 34$ & $0.4(2)$ \\
\hline $\mathrm{C} 5-\mathrm{C} 6-\mathrm{C} 7-\mathrm{C} 8$ & $0.0(3)$ & C32-C33-C34-C35 & $1.4(3)$ \\
\hline $\mathrm{C} 27-\mathrm{O} 3-\mathrm{C} 8-\mathrm{C} 9$ & $-1.0(3)$ & $\mathrm{C} 54-\mathrm{O} 6-\mathrm{C} 35-\mathrm{C} 36$ & $2.2(2)$ \\
\hline $\mathrm{C} 27-\mathrm{O} 3-\mathrm{C} 8-\mathrm{C} 7$ & $179.54(18)$ & $\mathrm{C} 54-\mathrm{O} 6-\mathrm{C} 35-\mathrm{C} 34$ & $-177.12(16)$ \\
\hline $\mathrm{C} 6-\mathrm{C} 7-\mathrm{C} 8-\mathrm{C} 9$ & $0.1(3)$ & $\mathrm{C} 33-\mathrm{C} 34-\mathrm{C} 35-\mathrm{C} 36$ & $-2.0(3)$ \\
\hline $\mathrm{C} 6-\mathrm{C} 7-\mathrm{C} 8-\mathrm{O} 3$ & $179.52(17)$ & $\mathrm{C} 33-\mathrm{C} 34-\mathrm{C} 35-\mathrm{O} 6$ & $177.34(15)$ \\
\hline $\mathrm{O} 3-\mathrm{C} 8-\mathrm{C} 9-\mathrm{C} 10$ & $179.69(16)$ & $\mathrm{O} 6-\mathrm{C} 35-\mathrm{C} 36-\mathrm{C} 37$ & $-178.40(14)$ \\
\hline $\mathrm{C} 7-\mathrm{C} 8-\mathrm{C} 9-\mathrm{C} 10$ & $-0.9(3)$ & $\mathrm{C} 34-\mathrm{C} 35-\mathrm{C} 36-\mathrm{C} 37$ & $0.9(2)$ \\
\hline $\mathrm{C} 8-\mathrm{C} 9-\mathrm{C} 10-\mathrm{C} 1$ & $179.38(15)$ & $\mathrm{C} 35-\mathrm{C} 36-\mathrm{C} 37-\mathrm{C} 32$ & $0.8(2)$ \\
\hline $\mathrm{C} 8-\mathrm{C} 9-\mathrm{C} 10-\mathrm{C} 5$ & $1.6(2)$ & $\mathrm{C} 35-\mathrm{C} 36-\mathrm{C} 37-\mathrm{C} 28$ & $-176.15(14)$ \\
\hline $\mathrm{C} 2-\mathrm{C} 1-\mathrm{C} 10-\mathrm{C} 9$ & $-178.42(15)$ & $\mathrm{C} 31-\mathrm{C} 32-\mathrm{C} 37-\mathrm{C} 36$ & $178.80(15)$ \\
\hline $\mathrm{C} 11-\mathrm{C} 1-\mathrm{C} 10-\mathrm{C} 9$ & $4.0(2)$ & $\mathrm{C} 33-\mathrm{C} 32-\mathrm{C} 37-\mathrm{C} 36$ & $-1.5(2)$ \\
\hline $\mathrm{C} 2-\mathrm{C} 1-\mathrm{C} 10-\mathrm{C} 5$ & $-0.7(2)$ & $\mathrm{C} 31-\mathrm{C} 32-\mathrm{C} 37-\mathrm{C} 28$ & $-4.0(2)$ \\
\hline $\mathrm{C} 11-\mathrm{C} 1-\mathrm{C} 10-\mathrm{C} 5$ & $-178.28(14)$ & $\mathrm{C} 33-\mathrm{C} 32-\mathrm{C} 37-\mathrm{C} 28$ & $175.70(14)$ \\
\hline $\mathrm{C} 6-\mathrm{C} 5-\mathrm{C} 10-\mathrm{C} 9$ & $-1.5(2)$ & $\mathrm{C} 29-\mathrm{C} 28-\mathrm{C} 37-\mathrm{C} 36$ & $-177.69(14)$ \\
\hline $\mathrm{C} 4-\mathrm{C} 5-\mathrm{C} 10-\mathrm{C} 9$ & $179.16(16)$ & $\mathrm{C} 38-\mathrm{C} 28-\mathrm{C} 37-\mathrm{C} 36$ & $5.4(2)$ \\
\hline $\mathrm{C} 6-\mathrm{C} 5-\mathrm{C} 10-\mathrm{C} 1$ & $-179.42(16)$ & $\mathrm{C} 29-\mathrm{C} 28-\mathrm{C} 37-\mathrm{C} 32$ & $5.3(2)$ \\
\hline $\mathrm{C} 4-\mathrm{C} 5-\mathrm{C} 10-\mathrm{C} 1$ & $1.3(2)$ & $\mathrm{C} 38-\mathrm{C} 28-\mathrm{C} 37-\mathrm{C} 32$ & $-171.61(13)$ \\
\hline $\mathrm{C} 2-\mathrm{C} 1-\mathrm{C} 11-\mathrm{O} 1$ & $-139.85(17)$ & $\mathrm{C} 29-\mathrm{C} 28-\mathrm{C} 38-\mathrm{O} 4$ & $132.66(16)$ \\
\hline $\mathrm{C} 10-\mathrm{C} 1-\mathrm{C} 11-\mathrm{O} 1$ & $37.8(2)$ & $\mathrm{C} 37-\mathrm{C} 28-\mathrm{C} 38-\mathrm{O} 4$ & $-50.4(2)$ \\
\hline $\mathrm{C} 2-\mathrm{C} 1-\mathrm{C} 11-\mathrm{C} 12$ & $40.1(2)$ & $\mathrm{C} 29-\mathrm{C} 28-\mathrm{C} 38-\mathrm{C} 39$ & $-49.21(19)$ \\
\hline $\mathrm{C} 10-\mathrm{C} 1-\mathrm{C} 11-\mathrm{C} 12$ & $-142.21(15)$ & $\mathrm{C} 37-\mathrm{C} 28-\mathrm{C} 38-\mathrm{C} 39$ & $127.73(15)$ \\
\hline $\mathrm{O} 1-\mathrm{C} 11-\mathrm{C} 12-\mathrm{C} 25$ & $-115.57(18)$ & $\mathrm{O} 4-\mathrm{C} 38-\mathrm{C} 39-\mathrm{C} 40$ & $-61.2(2)$ \\
\hline $\mathrm{C} 1-\mathrm{C} 11-\mathrm{C} 12-\mathrm{C} 25$ & $64.4(2)$ & $\mathrm{C} 28-\mathrm{C} 38-\mathrm{C} 39-\mathrm{C} 40$ & $120.68(15)$ \\
\hline $\mathrm{O} 1-\mathrm{C} 11-\mathrm{C} 12-\mathrm{C} 13$ & $64.3(2)$ & $\mathrm{O} 4-\mathrm{C} 38-\mathrm{C} 39-\mathrm{C} 52$ & $120.84(16)$ \\
\hline $\mathrm{C} 1-\mathrm{C} 11-\mathrm{C} 12-\mathrm{C} 13$ & $-115.69(17)$ & $\mathrm{C} 28-\mathrm{C} 38-\mathrm{C} 39-\mathrm{C} 52$ & $-57.30(19)$ \\
\hline $\mathrm{C} 25-\mathrm{C} 12-\mathrm{C} 13-\mathrm{C} 14$ & $176.42(15)$ & $\mathrm{C} 52-\mathrm{C} 39-\mathrm{C} 40-\mathrm{C} 45$ & $3.4(2)$ \\
\hline $\mathrm{C} 11-\mathrm{C} 12-\mathrm{C} 13-\mathrm{C} 14$ & $-3.4(2)$ & $\mathrm{C} 38-\mathrm{C} 39-\mathrm{C} 40-\mathrm{C} 45$ & $-174.59(13)$ \\
\hline $\mathrm{C} 25-\mathrm{C} 12-\mathrm{C} 13-\mathrm{C} 18$ & $-5.3(2)$ & $\mathrm{C} 52-\mathrm{C} 39-\mathrm{C} 40-\mathrm{C} 41$ & $-179.19(14)$ \\
\hline $\mathrm{C} 11-\mathrm{C} 12-\mathrm{C} 13-\mathrm{C} 18$ & $174.81(14)$ & $\mathrm{C} 38-\mathrm{C} 39-\mathrm{C} 40-\mathrm{C} 41$ & $2.9(2)$ \\
\hline $\mathrm{C} 12-\mathrm{C} 13-\mathrm{C} 14-\mathrm{C} 15$ & $-178.89(16)$ & $\mathrm{C} 39-\mathrm{C} 40-\mathrm{C} 41-\mathrm{C} 42$ & $-179.32(16)$ \\
\hline $\mathrm{C} 18-\mathrm{C} 13-\mathrm{C} 14-\mathrm{C} 15$ & $2.8(2)$ & $\mathrm{C} 45-\mathrm{C} 40-\mathrm{C} 41-\mathrm{C} 42$ & $-1.8(2)$ \\
\hline $\mathrm{C} 13-\mathrm{C} 14-\mathrm{C} 15-\mathrm{C} 16$ & $-1.1(3)$ & $\mathrm{C} 40-\mathrm{C} 41-\mathrm{C} 42-\mathrm{C} 43$ & $1.5(3)$ \\
\hline $\mathrm{C} 14-\mathrm{C} 15-\mathrm{C} 16-\mathrm{C} 17$ & $-0.8(3)$ & $\mathrm{C} 41-\mathrm{C} 42-\mathrm{C} 43-\mathrm{C} 44$ & $-0.2(3)$ \\
\hline $\mathrm{C} 15-\mathrm{C} 16-\mathrm{C} 17-\mathrm{C} 18$ & $0.8(3)$ & $\mathrm{C} 42-\mathrm{C} 43-\mathrm{C} 44-\mathrm{C} 45$ & $-0.5(3)$ \\
\hline $\mathrm{C} 12-\mathrm{C} 13-\mathrm{C} 18-\mathrm{C} 19$ & $0.7(2)$ & $\mathrm{C} 43-\mathrm{C} 44-\mathrm{C} 45-\mathrm{C} 46$ & $178.61(16)$ \\
\hline $\mathrm{C} 14-\mathrm{C} 13-\mathrm{C} 18-\mathrm{C} 19$ & $179.03(15)$ & $\mathrm{C} 43-\mathrm{C} 44-\mathrm{C} 45-\mathrm{C} 40$ & $0.1(2)$ \\
\hline $\mathrm{C} 12-\mathrm{C} 13-\mathrm{C} 18-\mathrm{C} 17$ & $178.94(14)$ & $\mathrm{C} 39-\mathrm{C} 40-\mathrm{C} 45-\mathrm{C} 46$ & $0.1(2)$ \\
\hline $\mathrm{C} 14-\mathrm{C} 13-\mathrm{C} 18-\mathrm{C} 17$ & $-2.7(2)$ & $\mathrm{C} 41-\mathrm{C} 40-\mathrm{C} 45-\mathrm{C} 46$ & $-177.49(14)$ \\
\hline $\mathrm{C} 16-\mathrm{C} 17-\mathrm{C} 18-\mathrm{C} 19$ & $179.17(17)$ & $\mathrm{C} 39-\mathrm{C} 40-\mathrm{C} 45-\mathrm{C} 44$ & $178.65(14)$ \\
\hline $\mathrm{C} 16-\mathrm{C} 17-\mathrm{C} 18-\mathrm{C} 13$ & $0.9(3)$ & $\mathrm{C} 41-\mathrm{C} 40-\mathrm{C} 45-\mathrm{C} 44$ & $1.0(2)$ \\
\hline $\mathrm{C} 13-\mathrm{C} 18-\mathrm{C} 19-\mathrm{C} 20$ & $3.5(3)$ & $\mathrm{C} 44-\mathrm{C} 45-\mathrm{C} 46-\mathrm{C} 47$ & $179.37(15)$ \\
\hline $\mathrm{C} 17-\mathrm{C} 18-\mathrm{C} 19-\mathrm{C} 20$ & $-174.67(15)$ & $\mathrm{C} 40-\mathrm{C} 45-\mathrm{C} 46-\mathrm{C} 47$ & $-2.1(2)$ \\
\hline
\end{tabular}




\begin{tabular}{llll}
$\mathrm{C} 18-\mathrm{C} 19-\mathrm{C} 20-\mathrm{C} 21$ & $175.16(16)$ & $\mathrm{C} 45-\mathrm{C} 46-\mathrm{C} 47-\mathrm{C} 48$ & $-179.13(14)$ \\
$\mathrm{C} 18-\mathrm{C} 19-\mathrm{C} 20-\mathrm{C} 25$ & $-3.1(2)$ & $\mathrm{C} 45-\mathrm{C} 46-\mathrm{C} 47-\mathrm{C} 52$ & $0.7(2)$ \\
$\mathrm{C} 19-\mathrm{C} 20-\mathrm{C} 21-\mathrm{C} 22$ & $-175.28(18)$ & $\mathrm{C} 46-\mathrm{C} 47-\mathrm{C} 48-\mathrm{C} 49$ & $176.31(15)$ \\
$\mathrm{C} 25-\mathrm{C} 20-\mathrm{C} 21-\mathrm{C} 22$ & $3.0(3)$ & $\mathrm{C} 52-\mathrm{C} 47-\mathrm{C} 48-\mathrm{C} 49$ & $-3.5(2)$ \\
$\mathrm{C} 20-\mathrm{C} 21-\mathrm{C} 22-\mathrm{C} 23$ & $-0.4(3)$ & $\mathrm{C} 47-\mathrm{C} 48-\mathrm{C} 49-\mathrm{C} 50$ & $0.2(2)$ \\
$\mathrm{C} 21-\mathrm{C} 22-\mathrm{C} 23-\mathrm{C} 24$ & $-2.3(3)$ & $\mathrm{C} 48-\mathrm{C} 49-\mathrm{C} 50-\mathrm{C} 51$ & $2.2(2)$ \\
$\mathrm{C} 22-\mathrm{C} 23-\mathrm{C} 24-\mathrm{C} 25$ & $2.5(3)$ & $\mathrm{C} 49-\mathrm{C} 50-\mathrm{C} 51-\mathrm{C} 52$ & $-1.1(2)$ \\
$\mathrm{C} 13-\mathrm{C} 12-\mathrm{C} 25-\mathrm{C} 24$ & $-171.16(15)$ & $\mathrm{C} 40-\mathrm{C} 39-\mathrm{C} 52-\mathrm{C} 51$ & $173.22(14)$ \\
$\mathrm{C} 11-\mathrm{C} 12-\mathrm{C} 25-\mathrm{C} 24$ & $8.7(2)$ & $\mathrm{C} 38-\mathrm{C} 39-\mathrm{C} 52-\mathrm{C} 51$ & $-8.8(2)$ \\
$\mathrm{C} 13-\mathrm{C} 12-\mathrm{C} 25-\mathrm{C} 20$ & $5.8(2)$ & $\mathrm{C} 40-\mathrm{C} 39-\mathrm{C} 52-\mathrm{C} 47$ & $-4.8(2)$ \\
$\mathrm{C} 11-\mathrm{C} 12-\mathrm{C} 25-\mathrm{C} 20$ & $-174.35(15)$ & $\mathrm{C} 38-\mathrm{C} 39-\mathrm{C} 52-\mathrm{C} 47$ & $173.18(13)$ \\
$\mathrm{C} 23-\mathrm{C} 24-\mathrm{C} 25-\mathrm{C} 12$ & $177.12(17)$ & $\mathrm{C} 50-\mathrm{C} 51-\mathrm{C} 52-\mathrm{C} 39$ & $179.70(14)$ \\
$\mathrm{C} 23-\mathrm{C} 24-\mathrm{C} 25-\mathrm{C} 20$ & $0.1(3)$ & $\mathrm{C} 50-\mathrm{C} 51-\mathrm{C} 52-\mathrm{C} 47$ & $-2.3(2)$ \\
$\mathrm{C} 19-\mathrm{C} 20-\mathrm{C} 25-\mathrm{C} 12$ & $-1.6(2)$ & $\mathrm{C} 46-\mathrm{C} 47-\mathrm{C} 52-\mathrm{C} 39$ & $2.8(2)$ \\
$\mathrm{C} 21-\mathrm{C} 20-\mathrm{C} 25-\mathrm{C} 12$ & $-179.88(15)$ & $\mathrm{C} 48-\mathrm{C} 47-\mathrm{C} 52-\mathrm{C} 39$ & $-177.42(13)$ \\
$\mathrm{C} 19-\mathrm{C} 20-\mathrm{C} 25-\mathrm{C} 24$ & $175.54(15)$ & $\mathrm{C} 46-\mathrm{C} 47-\mathrm{C} 52-\mathrm{C} 51$ & $-175.36(13)$ \\
$\mathrm{C} 21-\mathrm{C} 20-\mathrm{C} 25-\mathrm{C} 24$ & $-2.8(2)$ & $\mathrm{C} 48-\mathrm{C} 47-\mathrm{C} 52-\mathrm{C} 51$ & $4.5(2)$ \\
\hline
\end{tabular}

Hydrogen-bond geometry $\left(A,{ }^{\circ}\right)$

$C g 1, C g 2$ and $C g 3$ are the centroids of the $\mathrm{C} 20-\mathrm{C} 25, \mathrm{C} 1-\mathrm{C} 5 / \mathrm{C} 10$ and $\mathrm{C} 47-\mathrm{C} 52$ rings, respectively.

\begin{tabular}{lllll}
\hline$D-\mathrm{H} \cdots A$ & $D-\mathrm{H}$ & $\mathrm{H} \cdots A$ & $D \cdots A$ & $D-\mathrm{H} \cdots A$ \\
\hline $\mathrm{C} 7-\mathrm{H} 7 \cdots \mathrm{O} 4^{\mathrm{i}}$ & 0.95 & 2.54 & $3.419(3)$ & 154 \\
$\mathrm{C} 46-\mathrm{H} 46 \cdots \mathrm{O} 1$ & 0.95 & 2.57 & $3.2925(19)$ & 133 \\
$\mathrm{C} 49-\mathrm{H} 49 \cdots \mathrm{O} 4^{\mathrm{ii}}$ & 0.95 & 2.57 & $3.2515(19)$ & 128 \\
$\mathrm{C} 50-\mathrm{H} 50 \cdots 6^{\mathrm{iii}}$ & 0.95 & 2.56 & $3.368(2)$ & 143 \\
$\mathrm{C} 3-\mathrm{H} 3 \cdots C g 1^{\mathrm{iv}}$ & 0.95 & 2.68 & $3.557(2)$ & 153 \\
$\mathrm{C} 26-\mathrm{H} 26 A \cdots C g 2^{\mathrm{iv}}$ & 0.98 & 2.87 & $3.730(2)$ & 147 \\
$\mathrm{C} 30-\mathrm{H} 30 \cdots C g 3^{\mathrm{v}}$ & 0.95 & 2.71 & $3.602(2)$ & 157
\end{tabular}

Symmetry codes: (i) $x, y-1, z$; (ii) $x,-y+1 / 2, z-1 / 2$; (iii) $-x,-y,-z$; (iv) $-x+1,-y+2,-z$; (v) $-x,-y+1,-z$. 